\title{
Sampled-Data Exponential Synchronization of Markovian Jump Chaotic Lur'e Systems with Multiple Time Delays
}

\section{Te Yang}

Shandong University of Science and Technology

Zhen Wang ( $\boldsymbol{\sim}$ wangzhen@sdust.edu.cn )

Shandong University of Science and Technology https://orcid.org/0000-0003-1487-5089

\section{Xia Huang}

Shandong University of Science and Technology

Jianwei Xia

Liaocheng University

\section{Research Article}

Keywords: Markovian jump chaotic Lur'e systems, multiple time delays, aperiodic sampled-data controller, looped-functional, exponential stability

Posted Date: February 21st, 2022

DOI: https://doi.org/10.21203/rs.3.rs-1335598/v1

License: (c) (i) This work is licensed under a Creative Commons Attribution 4.0 International License. Read Full License 


\title{
Sampled-Data Exponential Synchronization of Markovian Jump Chaotic Lur'e Systems with Multiple Time Delays
}

\author{
Te Yang* ${ }^{*}$ hen Wang* Xia Huang ${ }^{\dagger}$ Jianwei Xia ${ }^{\ddagger}$
}

\begin{abstract}
This paper studies the mean square exponential synchronization control of the Markovian jump chaotic Lur'e systems (MJCLSs) with multiple time delays via aperiodic sampling. Firstly, by introducing the mode-dependent single/double integral terms and considering the system state at the sampling instant, a new mode-dependent Lyapunov functional based on the looped-functional is constructed. On the basis of the constructed functional and by estimating the expectation of the solution of MJCLSs with multiple time delays, an exponential synchronization criterion in the mean square sense is presented. Then, based on the obtained stability conditions, a design method for aperiodic sampled-data controller (ASDC) to ensure the exponential synchronization is proposed. Finally, the effectiveness of the developed method is demonstrated by the Chua's circuit model and a neural network model. Compared with the existing literature, an admissible larger upper bound of sampling period can be obtained.
\end{abstract}

Keywords: Markovian jump chaotic Lur'e systems; multiple time delays; aperiodic sampled-data controller; looped-functional; exponential stability

\section{Introduction}

The Lur'e system is a special kind of nonlinear systems proposed by Lur'e in the 1940s when studying the automatic pilot control of aircraft. It consists of a linear term and a sector-bounded nonlinear term. In [1-4], a large number of actual nonlinear systems such as positive rolling attractors, hyperchaotic systems, Chua's circuits and neural networks can all be simulated as chaotic Lur'e systems. However, the structure of an actual control system is often changed due to sudden external environment disturbances, changes in the coupling part of the subsystem and the random component failures and maintenance. In this case, we can use

\footnotetext{
${ }^{*}$ College of Mathematics and Systems Science, Shandong University of Science and Technology, Qingdao, Shandong, 266590, P. R. China. Email:yangte418@163.com (Te Yang), wangzhen@sdust.edu.cn (Zhen Wang, Corresponding author).

${ }^{\dagger}$ College of Electrical Engineering and Automation, Shandong University of Science and Technology, Qingdao, Shandong, 266590, P. R. China. Email:huangxia_qd@126.com (Xia Huang).

${ }^{\ddagger}$ School of Mathematics Science, Liaocheng University, Liaocheng, Shandong, 252000, P. R. China. Email:njustxjw@126.com (Jianwei Xia).
} 
the Markovian jump systems (MJSs) to simulate the dynamics. MJSs are widely used to simulate numerous actual engineering systems with unpredictable change in structure and parameters, such as aerospace systems, power systems and manufacturing systems. As we all know, the control problem of nonlinear MJSs has always been a hot spot in control field, especially the MJCLSs composed of linear and nonlinearities parts. Combining the random Lur'e-type Lyapunov functional and the implicit Markovian model method, sliding mode control problem was investigated for MJCLSs via event-triggered control in [5]. For the discrete-time MJCLSs with control saturation and exogenous $L_{2}$ type interference, the control synthesis problem of local stability and minimizing finite $L_{2}$ gain was solved in [6]. The model predictive control problem for a series of MJCLSs at the mercy of parameter uncertainties and input constraints was studied in [7]. The reduced-order and fullorder observers for MJCLSs differential inclusion system were designed in [8], which makes the corresponding error system exponentially stable.

Due to the wide application of digital controller in computer control systems and embedded control systems, networked control systems have been developed rapidly. As the theoretical basis, the stability study of sampled-data control system is particularly important [9-14]. As an important behavior of complex dynamic networks, synchronization has potential applications in the field of image processing, chemical reactions, communication safety and processes for storing and retrieving information. Since the pioneering work of synchronization in [15], the research on sampled-data synchronization of chaotic Lur'e systems has aroused the interest of many scholars. Based on the input delay approach, linear matrix inequalities and free-weighting matrix method, synchronization conditions in the global sense for chaotic Lur'e systems were proposed in $[16,17]$. A lower conservative design method for sampled-data controller and synchronization criteria were presented in [18] by constructing a Lyapunov functional that only requires the positiveness at sampling instants. Furthermore, most of the results consider the synchronization of chaotic delayed systems. Ref. [19, 20] used this type of functional proposed in Ref. [18] to consider the sampled-data synchronization problem of delayed chaotic Lur'e systems. A novel integral inequality approach and a novel Lyapunov functional was constructed by using more nonlinear information of the chaotic Lur'e delayed system, and less conservative stability conditions were presented in $[21,22] . H \infty$ synchronization and output feedback synchronization were studied in $[23,24]$ by constructing Lyapunov functionals. Quasi-synchronization of discrete-time Lur'etype switched systems with parameter mismatching and Lur'e networks with proportional delay were studied in $[25,26]$. The synchronization problem of uncertain delayed Lur'e system with random sampling based on Bernoulli distribution was addressed through a new non-fragile stochastic control method in [27]. Some less conservative synchronization conditions were presented based on the quantized sampled-data controller in $[28,29]$. Since neural networks and chaotic Lur'e systems have similar structures with nonlinear functions, much literature about sampled-data synchronization of Markovian jump neural networks (MJNNs) are of great significance to the research of sampled-data synchronization of MJCLSs. Considering coupled MJNNs with different forms of time delays, Ref. [30,31] addressed the stability and synchronization prob- 
lems. Considering the unknown transfer rate, and by constructing a mode-dependent Lyapunov functional, sampled-data synchronization of MJNNs was reported in [32,33]. Based on constructing mode-dependent one-sided and two-sided looped-functionals, the random sampled-data synchronization problem of MJNNs was studied in [34] and Ref. [34] concluded that two-sided looped-functionals has lower conservative. The $H \infty$ synchronization problems of MJNNs were studied by constructing a composite disturbance rejection controller and a mode-dependent synchronous controller in [35, 36], respectively.

Unlike such a wealth of research on sampled-data synchronization of MJNNs, the sampled-data synchronization of MJCLSs is less concerned, therefore, it needs further Consideration. Considering the event-trigger mechanism and the quantization effect, Ref. [37] studied the asynchronous control problem of MJCLSs. By considering partial accessibility and using hidden Markov model, Ref. [38] discussed the problem of asynchronous control of MJCLSs with limited time interval. By fully considering the probability distribution characteristics of packet loss, the random synchronization problem of a semi-MJCLSs with packet loss and multi-period sampling was studied in [39]. Through the above discussion, we can find that most of the chaotic Lur'e systems studied in the existing published papers are with single time-delay, and rarely literature considers the situation of multiple time delays. In addition, for MJCLSs, compared with the mode-independent Lyapunov functional, the mode-dependent Lyapunov functional is less conservative in many cases. However, in most studies of MJCLSs under aperiodic sampling, only the mode-dependent of the partial Lyapunov matrices is considered. However, when the single integral and double integral terms are introduced to construct the functional, the introduction of the mode-dependent matrices will inevitably bring about less conservative results. Furthermore, if one considers the use of sampled states to construct functionals, is it possible to construct mode-dependent functionals as well? Considering the above problems, the ASDC is further designed to guarantee exponential synchronization of the error system by constructing a lower conservative functional.

The following contributions have been presented:

1. A lemma to estimate the expectation of the solution of the MJCLSs with multiple time delays is proposed.

2. By introducing mode-dependent single/double integral terms to the Lyapunov functional, and considering mode-dependent information of the sampled-state, a innovative Lyapunov functional is constructed.

3. On the basis of the constructed Lyapunov functional and the improved integral inequality, the exponential synchronization criterion in the mean square sense is presented, and the ASDC is designed.

Notations: The following are the significant symbols used in this article. $\mathbb{R}:$ real numbers set; $\mathbb{R}^{n}: n$ dimensional Euclidean space; $\mathbb{R}^{m \times n}: m \times n$ real matrices set; $I(0)$ : identity (zero) matrix; $H^{-1}\left(H^{T}\right)$ : the inverse (transpose) of the matrix $H$; $\operatorname{diag}\{\ldots\}$ : block-diagonal matrix; $H>0(\geq 0)$ : real symmetric positivedefinite (positive-semidefinite) matrix; $\mathbb{E}\{\cdot\}:$ mathematical expectation; $\operatorname{col}\left\{\zeta_{1}, \zeta_{2}\right\}$ : the vector stacking $\zeta_{1}$ 
on the top of $\zeta_{2} ;\|A\|: \sup \{|A x|:|x|=1\}=\sqrt{\lambda_{\max }\left(A^{T} A\right)} ; *$ : symmetric components in a symmetric matrix; $\operatorname{sym}\{Q\}: Q+Q^{T} ; \lambda_{\min }(B)\left(\lambda_{\max }(B)\right):$ minimum (maximum) eigenvalues of matrix B; $\operatorname{Pr}\{N\}$ : Probability of event N.

\section{Problem Formulation}

Consider the master system as following:

$$
\mathscr{M}:\left\{\begin{array}{l}
\dot{x}(t)=\mathscr{A}_{r(t)} x(t)+\sum_{v=1}^{m} \mathscr{B}_{v, r(t)} x\left(t-d_{v}\right)+\mathscr{W}_{r(t)} \eta(\mathscr{D} x(t)), \\
q(t)=\mathscr{C}_{r(t)} x(t)
\end{array}\right.
$$

and the slave system

$$
\mathscr{N}:\left\{\begin{array}{l}
\dot{y}(t)=\mathscr{A}_{r(t)} y(t)+\sum_{v=1}^{m} \mathscr{B}_{v, r(t)} y\left(t-d_{v}\right)+\mathscr{W}_{r(t)} \eta(\mathscr{D} y(t))+u(t), \\
p(t)=\mathscr{C}_{r(t)} y(t),
\end{array}\right.
$$

where $u(t)=\mathscr{K}_{r(t)}\left(q\left(t_{k}\right)-p\left(t_{k}\right)\right), t_{k} \leq t<t_{k+1} . x(t), y(t) \in \mathbb{R}^{n}$ are state vectors, $q(t), p(t) \in \mathbb{R}^{l}$ are output vectors, $u(t) \in \mathbb{R}^{n}$ is control input, and $\mathscr{K}_{r(t)} \in \mathbb{R}^{n \times l}$ is control gain matrix. $d_{v}$ is the constant time-delay for $v=1,2, \ldots, m$. Assuming that $\eta(\cdot): R^{n} \rightarrow R^{n}$ is a diagonal nonlinearity and $\eta_{\sigma}(\cdot)$ belongs to the sector $\left[0, s_{\sigma}\right]$ for $\sigma=1,2, \ldots, n . \mathscr{A}_{r(t)}, \mathscr{B}_{v, r(t)}, \mathscr{W}_{r(t)}, \mathscr{D}$ and $\mathscr{C}_{r(t)}$ are known constant matrices. The sampling interval $h_{k}=t_{k+1}-t_{k}, k \geq 0$, the lower and upper bounds of the sampling interval is presented by $h_{m}$ and $h_{M}$, i.e., $0 \leq h_{m} \leq h_{k} \leq h_{M}$ with $t_{0}=0$.

Assuming $r(t)$ is a continuous-time markovian chain and it takes values from $\mathcal{N}=\{1,2, \ldots, N\}$ with generator $\Gamma \triangleq\left[r_{i j}\right]_{N \times N}$ given by

$$
\operatorname{Pr}\{r(t+l)=j \mid r(t)=i\}=\left\{\begin{array}{l}
r_{i j} l+o(l), i \neq j \\
1+r_{i i} l+o(l), i=j
\end{array}\right.
$$

here, $l>0, \lim _{l \rightarrow 0}(o(l) / l)=0 . r_{i j} \geq 0$ is transition rate from mode $i$ at time $t$ to mode $j$ at time $t+l$ if $i \neq j$ and $r_{i i}=-\sum_{j=1, j \neq i}^{N} r_{i j}$. To simplify, we denote $r(t)=i \in \mathcal{N}$. Let $\varsigma(t)=x(t)-y(t)$ is the synchronization error, we can express the error system like that:

$$
\dot{\zeta}(t)=\mathscr{A}_{i} \varsigma(t)+\sum_{v=1}^{m} \mathscr{B}_{v, i} \varsigma\left(t-d_{v}\right)+\mathscr{W}_{i} \xi(\mathscr{D} \varsigma(t), y(t))-\mathscr{K}_{i} \mathscr{C}_{i} \varsigma\left(t_{k}\right),
$$

here $\xi(\mathscr{D} \varsigma(t), y(t))=\eta(\mathscr{D}(\varsigma(t)+y(t)))-\eta\left(\mathscr{D}(y(t))\right.$. Let $\eta_{\sigma}(\cdot)$ pertains to sector $\left[0, s_{\sigma}\right]$, for any $\sigma=$ $1,2, \ldots, n$, it is easy to find like this

$$
0 \leq \frac{\xi_{\sigma}\left(d_{\sigma}^{T} \varsigma, y\right)}{d_{\sigma}^{T} \varsigma}=\frac{\eta_{\sigma}\left[d_{\sigma}^{T}(\varsigma+y)\right]-\eta_{\sigma}\left(d_{\sigma}^{T} \varsigma\right)}{d_{\sigma}^{T} \varsigma} \leq s_{\sigma},
$$


where $d_{\sigma}^{T}$ represents the $\sigma$ th row vector of $\mathscr{D}$. From [19], for any matrices $\mathscr{V}_{\rho, i}=\operatorname{diag}\left\{v_{\rho, 1, i}, v_{\rho, 2, i}, \ldots, v_{\rho, n, i}\right\} \geq$ $0, \rho=1,2$, the inequalities can be presented like this:

$$
\varsigma^{T}(t) \mathscr{D}^{T} \mathscr{S}{ }_{\rho, i} \xi(\mathscr{D} \varsigma(t), y(t))-\xi(\mathscr{D} \varsigma(t), y(t))^{T} \mathscr{V}_{\rho, i} \xi(\mathscr{D} \varsigma(t), y(t)) \geq 0
$$

where $\mathscr{S}=\operatorname{diag}\left\{s_{1}, s_{2}, \ldots, s_{n}\right\}$.

The objective of this research is to develop a new design method for ASDC via a novel Lyapunov functional to make the master system $\mathscr{M}$ and the slave system $\mathscr{N}$ mean square exponential synchronize. Before giving the results of stability analysis, we supply a stability definition and two useful lemmas.

Definition 1. [40] The synchronization error system (3) is exponentially stable in the mean square sense, if there exists a decay coefficient $\beta>0$ and a decay rate $\alpha>0$ such that

$$
\mathbb{E}\left\{\|\varsigma(t)\|^{2}\right\} \leq \beta e^{-2 \alpha t} \mathbb{E}\left\{\left\|\varsigma_{0}\right\|_{c}^{2}\right\}, \quad \forall t \geq 0,
$$

where $\left\|\varsigma_{0}\right\|_{c}^{2}=\sup _{-\chi \leq \theta \leq 0}\left\{\|\varsigma(\theta)\|^{2}+\|\dot{\zeta}(\theta)\|^{2}\right\}$, and $\chi=\max \left\{d_{v}, h_{M}\right\}, v=1,2, \ldots, m$.

Lemma 1. Concerning system (3), the following inequality holds:

$$
\mathbb{E}\left\{|\varsigma(t)|^{2}\right\} \leq \theta_{1} \mathbb{E}\left\{\left\|\varsigma\left(t_{k}\right)\right\|^{2}\right\}+\mathbb{E}\left\{\sum_{v=1}^{m} \int_{t_{k}-d_{v}}^{t_{k}} \theta_{2} \varepsilon\|\varsigma(s)\|^{2} d s\right\},
$$

where

$$
\begin{aligned}
\theta_{1} & =\left(5+5 h_{M}^{2}\left\|\mathscr{K}_{i} \mathscr{C}_{i}\right\|^{2}\right) \varepsilon, \\
\theta_{2} & =5 h_{M} m\left\|\mathscr{B}_{v, i}\right\|^{2}, \\
\varepsilon & =e^{5 h_{M}^{2}\left\|\mathscr{A}_{i}\right\|^{2}+5 h_{M}^{2} \sum_{v=1}^{m} m\left\|\mathscr{B}_{v, i}\right\|^{2}+5 h_{M}^{2}\left\|\mathscr{W}_{i}\right\|^{2}\|\mathscr{S} \mathscr{D}\|^{2}} .
\end{aligned}
$$

Proof. Integrating system (3) from $t_{k}$ to $t$ leads to

$$
\begin{aligned}
\|\varsigma(t)\| \leq & \left\|\varsigma\left(t_{k}\right)\right\|+\left\|\int_{t_{k}}^{t} \mathscr{A}_{i} \varsigma(s) d s\right\|+\left\|\int_{t_{k}}^{t} \mathscr{K}_{i} \mathscr{C}_{i} \varsigma\left(t_{k}\right) d s\right\| \\
& +\left\|\int_{t_{k}}^{t} \sum_{v=1}^{m} \mathscr{B}_{v, i} \varsigma\left(s-d_{v}\right) d s\right\|+\left\|\int_{t_{k}}^{t} \mathscr{W}_{i} \xi(\mathscr{D} \varsigma(s), y(s)) d s\right\| .
\end{aligned}
$$

In addition, from Cauchy-Schwarz inequality, we can easily obtain that

$$
\begin{aligned}
\|\varsigma(t)\|^{2} \leq & 5\left\|\varsigma\left(t_{k}\right)\right\|^{2}+5\left\|\int_{t_{k}}^{t} \mathscr{A}_{i} \varsigma(s) d s\right\|^{2}+5\left\|\int_{t_{k}}^{t} \sum_{v=1}^{m} \mathscr{B}_{v, i} \varsigma\left(s-d_{v}\right) d s\right\|^{2} \\
& +5\left\|\int_{t_{k}}^{t} \mathscr{W}_{i} \xi\left(\mathscr{D}_{\varsigma}(s), y(s)\right) d s\right\|^{2}+5\left\|\int_{t_{k}}^{t} \mathscr{K}_{i} \mathscr{C}_{i} \varsigma\left(t_{k}\right) d s\right\|^{2},
\end{aligned}
$$

and

$$
\mathbb{E}\left\{\|\varsigma(t)\|^{2}\right\} \leq 5 \mathbb{E}\left\{\left\|\varsigma\left(t_{k}\right)\right\|\right\}^{2}+5 h_{M} \mathbb{E}\left\{\int_{t_{k}}^{t}\left\|\mathscr{A}_{i} \varsigma(s)\right\|^{2} d s\right\}+5 h_{M} \mathbb{E}\left\{\int_{t_{k}}^{t}\left\|\sum_{v=1}^{m} \mathscr{B}_{v, i} \varsigma\left(s-d_{v}\right)\right\|^{2} d s\right\}
$$




$$
+5 h_{M} \mathbb{E}\left\{\int_{t_{k}}^{t}\left\|\mathscr{W}_{i} \xi(\mathscr{D} \varsigma(s), y(s))\right\|^{2} d s\right\}+5 h_{M} \mathbb{E}\left\{\int_{t_{k}}^{t}\left\|\mathscr{K}_{i} \mathscr{C}_{i} \varsigma\left(t_{k}\right)\right\|^{2} d s\right\} .
$$

It can be found from (4) that

$$
\|\xi(\mathscr{D} \varsigma(t), y(t))\|^{2} \leq\|\mathscr{S} \mathscr{D}\|^{2}\|\varsigma(t)\|^{2}
$$

and

$$
\begin{aligned}
& 5 h_{M} \mathbb{E}\left\{\int_{t_{k}}^{t}\left\|\sum_{v=1}^{m} \mathscr{B}_{v, i} \varsigma\left(s-d_{v}\right)\right\|^{2} d s\right\} \\
& \leq 5 h_{M} \mathbb{E}\left\{\sum_{v=1}^{m} \int_{t_{k}}^{t} m\left\|\mathscr{B}_{v, i} \varsigma\left(s-d_{v}\right)\right\|^{2} d s\right\} \\
& \leq 5 h_{M} m \mathbb{E}\left\{\sum_{v=1}^{m}\left\|\mathscr{B}_{v, i}\right\|^{2} \int_{t_{k}-d_{v}}^{t-d_{v}}\|\varsigma(s)\|^{2} d s\right\} \\
& \leq 5 h_{M} m \mathbb{E}\left\{\sum_{v=1}^{m}\left\|\mathscr{B}_{v, i}\right\|^{2} \int_{t_{k}-d_{v}}^{t_{k}}\|\varsigma(s)\|^{2} d s\right\}+5 h_{M} m \mathbb{E}\left\{\sum_{v=1}^{m}\left\|\mathscr{B}_{v, i}\right\|^{2} \int_{t_{k}}^{t}\|\varsigma(s)\|^{2} d s\right\} .
\end{aligned}
$$

Thence, we can obtain

$$
\begin{aligned}
\mathbb{E}\left\{\|\varsigma(t)\|^{2}\right\} \leq & 5 \mathbb{E}\left\{\left\|\varsigma\left(t_{k}\right)\right\|\right\}^{2}+5 h_{M}\left\|\mathscr{A}_{i}\right\|^{2} \mathbb{E}\left\{\int_{t_{k}}^{t}\|\varsigma(s)\|^{2} d s\right\} \\
& +5 h_{M} m \sum_{v=1}^{m}\left\|\mathscr{B}_{v, i}\right\|^{2} \mathbb{E}\left\{\int_{t_{k}-d_{v}}^{t_{k}}\|\varsigma(s)\|^{2} d s\right\}+5 h_{M} m \sum_{v=1}^{m}\left\|\mathscr{B}_{v, i}\right\|^{2} \mathbb{E}\left\{\int_{t_{k}}^{t}\|\varsigma(s)\|^{2} d s\right\} \\
& +5 h_{M}\left\|\mathscr{W}_{i}\right\|^{2}\|\mathscr{S} \mathscr{D}\|^{2} \mathbb{E}\left\{\int_{t_{k}}^{t}\|\varsigma(s)\|^{2} d s\right\}+5 h_{M}^{2}\left\|\mathscr{K}_{i} \mathscr{C}_{i}\right\|^{2} \mathbb{E}\left\{\left\|\varsigma\left(t_{k}\right)\right\|\right\}^{2} \\
\leq & \left(5+5 h_{M}^{2}\left\|\mathscr{K}_{i} \mathscr{C}_{i}\right\|^{2}\right) \mathbb{E}\left\{\left\|\varsigma\left(t_{k}\right)\right\|^{2}\right\}+\left(5 h_{M}\left\|\mathscr{A}_{i}\right\|^{2}+5 h_{M} m \sum_{v=1}^{m}\left\|\mathscr{B}_{v, i}\right\|^{2}\right. \\
& \left.+5 h_{M}\left\|\mathscr{W}_{i}\right\|^{2}\|\mathscr{S} \mathscr{D}\|^{2}\right) \mathbb{E}\left\{\int_{t_{k}}^{t}\|\varsigma(s)\|^{2} d s\right\}+5 h_{M} m \sum_{v=1}^{m}\left\|\mathscr{B}_{v, i}\right\|^{2} \mathbb{E}\left\{\int_{t_{k}-d_{v}}^{t_{k}}\|\varsigma(s)\|^{2} d s\right\} .
\end{aligned}
$$

From the Gronwall inequality, we can obtain (7).

Remark 1. For error system (3), the estimation of expectation of the system solution is presented by Lemma 1, which plays an important role in analysis of the mean square exponential stability.

Lemma 2. [41] For given symmetric matrices $R>0$ and $N_{1}, N_{2}, N_{3}$, the following inequality satisfies:

$$
-\int_{a}^{b} \dot{\zeta}^{T}(t) R \dot{\varsigma}(t) d s \leq \varpi^{T} \Omega \varpi
$$

where

$$
\begin{aligned}
& \Omega=\tau\left(N_{1} R^{-1} N_{1}^{T}+\frac{1}{3} N_{2} R^{-1} N_{2}^{T}+\frac{1}{5} N_{3} R^{-1} N_{3}^{T}\right)+\operatorname{Sym}\left\{N_{1} \Pi_{1}+N_{2} \Pi_{2}+N_{3} \Pi_{3}\right\}, \\
& \Pi_{1}=\check{e}_{1}-\check{e}_{2}, \quad \Pi_{2}=\check{e}_{1}+\check{e}_{2}-2 \check{e}_{3}, \quad \Pi_{3}=\check{e}_{1}-\check{e}_{2}-6 \check{e}_{3}+6 \check{e}_{4}, \quad \tau=a-b, \\
& \varpi=\left[\begin{array}{lll}
x^{T}(b), \quad x^{T}(a), \quad \frac{1}{\tau} \int_{a}^{b} x^{T}(s) d s, \quad \frac{2}{\tau^{2}} \int_{a}^{b} \int_{a}^{s} x^{T}(u) d u d s
\end{array}\right] \\
& \check{e}_{\check{\iota}}=\left[\begin{array}{lll}
0_{n \times(\check{\iota}-1) n}, \quad I_{n \times n}, \quad 0_{n \times(4-\check{\iota}) n}
\end{array}\right], \quad \check{\iota}=1,2,3,4 .
\end{aligned}
$$




\section{Stability analysis and controller design}

In this section, a mode-dependent two-sided looped-functional was constructed. Using the novel functional, we discuss the exponentially synchronization in the mean square sense for MJCLSs with multiple time delays. The ASDC design scheme for error system is proposed. For simplicity, we use nomenclature for vectors and matrices in the following.

$$
\begin{aligned}
& \omega_{1, v}=\frac{1}{d_{v}} \int_{t-d_{v}}^{t} \varsigma(s) d s, \quad \omega_{2, v}=\frac{2}{d_{v}{ }^{2}} \int_{t-d_{v}}^{t} \int_{t-d_{v}}^{s} \varsigma(u) d u d s, \quad \alpha_{1, v}=\frac{\mathrm{e}^{2 \alpha d_{v}}-1}{2 \alpha}, \\
& \alpha_{2, v}=\frac{\mathrm{e}^{2 \alpha d_{v}}-1-2 \alpha d_{v}}{4 \alpha^{2}}, \quad \alpha_{3, v}=\frac{\mathrm{e}^{2 \alpha d_{v}}-1-2 \alpha d_{v}+2 \alpha^{2} d_{v}{ }^{2}}{8 \alpha^{3}}, \quad \alpha_{4}=\frac{\mathrm{e}^{-2 \alpha h_{M}}-1+2 \alpha h_{M}}{4 \alpha^{2}},
\end{aligned}
$$

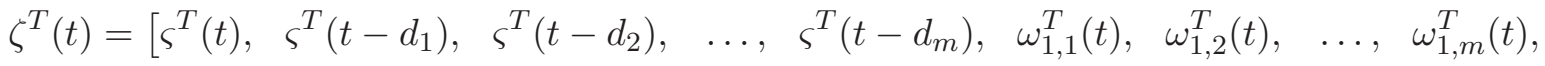

$$
\begin{aligned}
& \left.\omega_{2,1}^{T}(t), \quad \omega_{2,2}^{T}(t), \quad \ldots, \quad \omega_{2, m}^{T}(t), \quad \varsigma^{T}\left(t_{k}\right), \quad \varsigma^{T}\left(t_{k+1}\right), \quad \dot{\zeta}^{T}(t), \quad \xi^{T}(\mathscr{D} \varsigma(t), y(t))\right], \\
& \zeta_{1}^{T}(t)=\left[\left(t_{k+1}-t\right) \mu_{1}^{T}(t), \quad\left(t-t_{k}\right) \mu_{2}^{T}(t)\right], \\
& \zeta_{2}^{T}(t)=\left[\mu_{1}^{T}(t), \quad \mu_{2}^{T}(t)\right], \quad \zeta_{3}^{T}=\left[\varsigma^{T}\left(t_{k}\right), \quad \varsigma^{T}\left(t_{k+1}\right)\right], \\
& \mu_{1}(t)=\varsigma(t)-\varsigma\left(t_{k}\right), \quad \mu_{2}(t)=\varsigma(t)-\varsigma\left(t_{k+1}\right), \\
& e_{\iota}=\left[\begin{array}{lll}
0_{n \times(\iota-1) n}, & I_{n \times n}, & 0_{n \times(3 m+5-\iota) n}
\end{array}\right], \quad \iota=1,2, \ldots, 3 m+5 .
\end{aligned}
$$

Theorem 1. Given scalars $\alpha>0, \delta$ and $\gamma$, the slave system (2) is exponentially synchronization in the mean square sense to the master system (1) if there exist positive definite matrices $P_{i}, Q_{v, i}, Q_{v}, \mathscr{R}_{v, i}, \mathscr{R}_{v}$, $U_{i}, U, Z_{i}$, matrices $X_{i}, Y_{i}, S_{i}, M_{1, v, i}, M_{2, v, i}, M_{3, v, i} G_{i} L_{i}$, and diagonal matrices $\mathscr{V}_{1, i}>0, \mathscr{V}_{2, i}>0$ such that following conditions hold for $h_{k} \in\left\{h_{m}, h_{M}\right\}, \forall i \in \mathcal{N}, v=1,2, \ldots, m$ :

$$
\hat{\Xi}_{1}\left(h_{k}\right)=\left[\begin{array}{cccccc}
\Phi_{1}+\Phi_{2}+h_{k} \Phi_{3} & \sqrt{d_{1}} \widetilde{\Xi}_{1} & \sqrt{d_{2}} \widetilde{\Xi}_{2} & \sqrt{d_{2}} \widetilde{\Xi}_{3} & \ldots & \sqrt{d_{m}} \widetilde{\Xi}_{m} \\
* & \widetilde{R}_{1, i} & 0 & 0 & \ldots & 0 \\
* & * & \widetilde{R}_{2, i} & 0 & \ldots & 0 \\
* & * & * & \widetilde{R}_{3, i} & \ldots & 0 \\
\vdots & \vdots & \vdots & \vdots & \ddots & \vdots \\
* & * & * & * & \ldots & \widetilde{R}_{m, i}
\end{array}\right]<0,
$$




$$
\begin{array}{r}
e^{2 \alpha d_{v}} \sum_{j=1}^{N} r_{i j} R_{v, j}-R_{v}<0, \\
\sum_{j=1}^{N} r_{i j} U_{j}-U<0
\end{array}
$$

where

$$
\begin{aligned}
& \pi_{1}=\left[e_{3 m+2}^{T}-e_{1}^{T}, \quad e_{1}^{T}-e_{3 m+3}^{T}\right]^{T}, \quad \pi_{2}=\left[\begin{array}{ll}
e_{1}^{T}-e_{3 m+2}^{T}, & e_{1}^{T}-e_{3 m+3}^{T}
\end{array}\right]^{T}, \quad \pi_{3}=\left[\begin{array}{ll}
e_{3 m+2}^{T}, & e_{3 m+3}^{T}
\end{array}\right]^{T}, \\
& \pi_{4}=\left[\begin{array}{ll}
e_{3 m+4}^{T}, & e_{3 m+4}^{T}
\end{array}\right]^{T}, \quad \pi_{5}=\left[\begin{array}{ll}
e_{3 m+4}^{T}, & 0
\end{array}\right]^{T}, \quad \pi_{6}=\left[\begin{array}{ll}
0, & e_{3 m+4}^{T}
\end{array}\right]^{T}, \quad \pi_{7, v}=e_{1}-e_{v+1}, \\
& \pi_{8, v}=e_{1}+e_{v+1}-2 e_{m+v+1}, \quad \pi_{9, v}=e_{1}-e_{v+1}-6 e_{m+v+1}+6 e_{2 m+v+1}, \quad \pi_{10}=\left[e_{1}^{T}-e_{3 m+2}^{T}, \quad 0\right]^{T},
\end{aligned}
$$

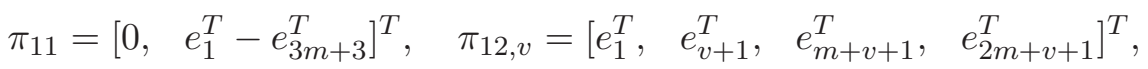

$$
\begin{aligned}
& \pi_{13}=\left[\begin{array}{ll}
e_{3 m+2}^{T}, & e_{3 m+3}^{T}
\end{array}\right]^{T}, \quad \pi_{14}=e_{1}-e_{3 m+2}, \quad \pi_{15}=e_{1}-e_{3 m+3}, \\
& \Phi_{1}=e_{1}^{T}\left[2 \alpha P_{i}+\left(\sum_{j=1}^{N} r_{i j}\right) P_{j}+\sum_{v=1}^{m} e^{2 \alpha d_{v}} Q_{v, i}+\sum_{v=1}^{m} \alpha_{1, v} Q_{v}\right] e_{1}+e_{3 m+4}^{T}\left(U_{i}+h_{M} U\right) e_{3 m+4} \\
& -\sum_{v=1}^{m} e_{v+1}^{T} Q_{v, i} e_{v+1}+e_{3 m+4}^{T}\left(\sum_{v=1}^{m} \alpha_{1, v} \mathscr{R}_{v, i}+\sum_{v=1}^{m} \alpha_{2, v} \mathscr{R}_{v}\right) e_{3 m+4}+\pi_{13}^{T}\left[2 \alpha h_{M}^{2} Z_{i}+h_{M}^{2}\left(\sum_{j=1}^{N} r_{i j} Z_{j}\right)\right] \pi_{13} \\
& +\operatorname{sym}\left\{e_{1}^{T} P_{i} e_{3 m+4}+\pi_{1}^{T}\left(Y_{i} \pi_{2}+S_{i} \pi_{3}\right)+\pi_{14}^{T}\left[2 \alpha X_{i}+\left(\sum_{j=1}^{N} r_{i j} X_{j}\right)\right] \pi_{15}+e_{3 m+4}^{T} X_{i} \pi_{15}+\pi_{14}^{T} X_{i} e_{3 m+4}\right. \\
& +\left(\delta e_{1}^{T} G_{i}+e_{3 m+4}^{T} G_{i}+\gamma e_{3 m+2}^{T} G_{i}\right)\left(-e_{3 m+4}+\mathscr{A}_{i} e_{1}+\sum_{v=1}^{m} \mathscr{B}_{v, i} e_{v+1}+\mathscr{W}_{i} e_{3 m+5}-\mathscr{K}_{i} \mathscr{C}_{i} e_{3 m+2}\right) \\
& \left.+\sum_{v=1}^{m} \pi_{12, v}^{T}\left(M_{1, v, i} \pi_{7, v}+M_{2, v, i} \pi_{8, v}+M_{3, v, i} \pi_{9, v}\right)\right\}, \\
& \Phi_{2}=\operatorname{sym}\left\{e_{1}^{T} \mathscr{D}^{T} \mathscr{S} \mathscr{V}_{1, i} e_{3 m+5}\right\}-2 e_{3 m+5}^{T} \mathscr{V}_{1, i} e_{3 m+5}, \\
& \Phi_{3}=\operatorname{sym}\left\{\left(\pi_{5}^{T}+2 \alpha \pi_{10}^{T}\right)\left(Y_{i} \pi_{2}+S_{i} \pi_{3}\right)+\pi_{10}^{T} Y_{i} \pi_{4}+\pi_{10}^{T}\left[\left(\sum_{j=1}^{N} r_{i j} Y_{j}\right) \pi_{2}+\left(\sum_{j=1}^{N} r_{i j} S_{j}\right) \pi_{3}\right]\right\}+\pi_{13}^{T} Z_{i} \pi_{13}, \\
& \Phi_{4}=\operatorname{sym}\left\{e_{1}^{T} \mathscr{D}^{T} \mathscr{S} \mathscr{V}_{2, i} e_{3 m+5}\right\}-2 e_{3 m+5}^{T} \mathscr{N}_{2, i} e_{3 m+5}, \\
& \Phi_{5}=\operatorname{sym}\left\{\left(\pi_{6}^{T}+2 \alpha \pi_{11}^{T}\right)\left(Y_{i} \pi_{2}+S_{i} \pi_{3}\right)+\pi_{11}^{T} Y_{i} \pi_{4}+\pi_{11}^{T}\left[\left(\sum_{j=1}^{N} r_{i j} Y_{j}\right) \pi_{2}+\left(\sum_{j=1}^{N} r_{i j} S_{j}\right) \pi_{3}\right]\right\}-\pi_{13}^{T} Z_{i} \pi_{13}, \\
& \widetilde{\Xi}_{v}=\left[\pi_{12, v}^{T} M_{1, v, i}, \quad \pi_{12, v}^{T} M_{2, v, i}, \quad \pi_{12, v}^{T} M_{3, v, i}\right], \\
& \widetilde{\mathscr{R}}_{v, i}=\operatorname{diag}\left\{-\mathscr{R}_{v, i}, \quad-3 \mathscr{R}_{v, i}, \quad-5 \mathscr{R}_{v, i}\right\} .
\end{aligned}
$$

Furthermore, the ASDC gain matrix in (3) is

$$
\mathscr{K}_{i}=G_{i}^{-1} L_{i} .
$$

Proof. Constructing mode-dependent two-sided looped-functional for the system (3)

$$
\mathcal{V}(\varsigma(t), i, t)=\sum_{\epsilon=1}^{7} \mathcal{V}_{\epsilon}(\varsigma(t), i, t),
$$


with

$$
\begin{aligned}
& \mathcal{V}_{1}(\varsigma(t), i, t)=\mathrm{e}^{2 \alpha t} \varsigma^{T}(t) P_{i} \varsigma(t), \\
& \mathcal{V}_{2}(\varsigma(t), i, t)=\sum_{v=1}^{m} \int_{t-d_{v}}^{t} \mathrm{e}^{2 \alpha\left(s+d_{v}\right)} \varsigma^{T}(s) Q_{v, i} \varsigma(s) d s+\sum_{v=1}^{m} \int_{-d_{v}}^{0} \int_{t+\theta}^{t} \mathrm{e}^{2 \alpha(s-\theta)} \varsigma^{T}(s) Q_{v} \varsigma(s) d s d \theta, \\
& \mathcal{V}_{3}(\varsigma(t), i, t)=\sum_{v=1}^{m} \int_{-d_{v}}^{0} \int_{t+\theta}^{t} \mathrm{e}^{2 \alpha(s-\theta)} \dot{\varsigma}^{T}(s) \mathscr{R}_{v, i} \dot{\zeta}(s) d s d \theta+\sum_{v=1}^{m} \int_{-d_{v}}^{0} \int_{\theta}^{0} \int_{t+\beta}^{t} \mathrm{e}^{2 \alpha(s-\beta)} \dot{\varsigma}^{T}(s) \mathscr{R}_{v} \dot{\zeta}(s) d s d \beta d \theta, \\
& \mathcal{V}_{4}(\varsigma(t), i, t)=\int_{t_{k}}^{t} \mathrm{e}^{2 \alpha s} \dot{\varsigma}^{T}(s) U_{i} \dot{\varsigma}(s) d s+\int_{-h_{M}}^{0} \int_{t+\theta}^{t} \mathrm{e}^{2 \alpha s} \dot{\varsigma}^{T}(s) U \dot{\zeta}(s) d s d \theta \\
& \mathcal{V}_{5}(\varsigma(t), i, t)=2 \mathrm{e}^{2 \alpha t} \mu_{1}^{T}(t) X_{i} \mu_{2}(t), \\
& \mathcal{V}_{6}(\varsigma(t), i, t)=\left(t_{k+1}-t\right)\left(t-t_{k}\right) \mathrm{e}^{2 \alpha t} \zeta_{3}^{T} Z_{i} \zeta_{3}, \\
& \mathcal{V}_{7}(\varsigma(t), i, t)=2 \mathrm{e}^{2 \alpha t} \zeta_{1}^{T}(t)\left[Y_{i} \zeta_{2}(t)+S_{i} \zeta_{3}\right] .
\end{aligned}
$$

Let $\mathcal{L}$ be the weak infinitesimal operator of the random process $\{\varsigma(t), i, t \geqslant 0\}$ acting on $\mathcal{V}(\varsigma(t), i, t)$. Then, for any $i \in \mathcal{N}$, calculating $\mathcal{L} \mathcal{V}(\varsigma(t), i, t)$ of $(3)$ gives

$$
\begin{aligned}
\mathcal{L} \mathcal{V}_{1}(\varsigma(t), i, t) & =2 \alpha \mathrm{e}^{2 \alpha t} \varsigma^{T}(t) P_{i} \varsigma(t)+2 \mathrm{e}^{2 \alpha t} \varsigma^{T}(t) P_{i} \dot{\varsigma}(t)+\mathrm{e}^{2 \alpha t} \varsigma^{T}(t)\left(\sum_{j=1}^{N} r_{i j} P_{j}\right) \varsigma(t) \\
& =\mathrm{e}^{2 \alpha t}\left\{\zeta^{T}(t)\left[e_{1}^{T}\left(2 \alpha P_{i}+\left(\sum_{j=1}^{N} r_{i j} P_{j}\right)\right) e_{1}+2 e_{1}^{T} P_{i} e_{3 m+4}\right] \zeta(t)\right\},
\end{aligned}
$$

and

$$
\begin{aligned}
\mathcal{L} \mathcal{V}_{2}(\varsigma(t), i, t)= & \mathrm{e}^{2 \alpha t}\left\{\varsigma^{T}(t)\left[\sum_{v=1}^{m} \mathrm{e}^{2 \alpha d_{v}} Q_{v, i}+\sum_{v=1}^{m} \alpha_{1, v} Q_{v}\right] \varsigma(t)-\sum_{v=1}^{m} \varsigma^{T}\left(t-d_{v}\right) Q_{v, i} \varsigma\left(t-d_{v}\right)\right\} \\
& -\mathrm{e}^{2 \alpha t} \sum_{v=1}^{m} \int_{t-d_{v}}^{t} \varsigma^{T}(s) Q_{v} \varsigma(s) d s+\sum_{v=1}^{m} \int_{t-d_{v}}^{t} \mathrm{e}^{2 \alpha\left(s+d_{v}\right)} \varsigma^{T}(s)\left(\sum_{j=1}^{N} r_{i j} Q_{v, j}\right) \varsigma(s) d s .
\end{aligned}
$$

It follows from (11) and $Q_{v}>0$ that

$$
\sum_{v=1}^{m} \int_{t-d_{v}}^{t} \mathrm{e}^{2 \alpha\left(s+d_{v}\right)} \varsigma^{T}(s)\left(\sum_{j=1}^{N} r_{i j} Q_{v, j}\right) \varsigma(s) d s \leq \sum_{v=1}^{m} \int_{t-d_{v}}^{t} \mathrm{e}^{2 \alpha s} \varsigma^{T}(s) Q_{v} \varsigma(s) d s \leq \mathrm{e}^{2 \alpha t} \sum_{v=1}^{m} \int_{t-d_{v}}^{t} \varsigma^{T}(s) Q_{v} \varsigma(s) d s,
$$

that is, from (11), there is the following inequality

$$
\sum_{v=1}^{m} \int_{t-d_{v}}^{t} \mathrm{e}^{2 \alpha\left(s+d_{v}\right)} \varsigma^{T}(s)\left(\sum_{j=1}^{N} r_{i j} Q_{v, j}\right) \varsigma(s) d s-\mathrm{e}^{2 \alpha t} \sum_{v=1}^{m} \int_{t-d_{v}}^{t} \varsigma^{T}(s) Q_{v} \varsigma(s) d s \leq 0 .
$$

Therefore,

$$
\begin{aligned}
\mathcal{L} \mathcal{V}_{2}(\varsigma(t), i, t) & \leq \mathrm{e}^{2 \alpha t}\left\{\varsigma^{T}(t)\left[\sum_{v=1}^{m} \mathrm{e}^{2 \alpha d_{v}} Q_{v, i}+\sum_{v=1}^{m} \alpha_{1, v} Q_{v}\right] \varsigma(t)-\sum_{v=1}^{m} \varsigma^{T}\left(t-d_{v}\right) Q_{v, i} \varsigma\left(t-d_{v}\right)\right\} \\
& =\mathrm{e}^{2 \alpha t} \zeta^{T}(t)\left[e_{1}^{T}\left(\sum_{v=1}^{m} \mathrm{e}^{2 \alpha d_{v}} Q_{v, i}+\sum_{v=1}^{m} \alpha_{1, v} Q_{v}\right) e_{1}-\sum_{v=1}^{m} e_{v+1}^{T} Q_{v, i} e_{v+1}\right] \zeta(t) .
\end{aligned}
$$




$$
\begin{aligned}
\mathcal{L} \mathcal{V}_{3}(\varsigma(t), i, t)= & \mathrm{e}^{2 \alpha t}\left[\sum_{v=1}^{m} \alpha_{1, v} \dot{\zeta}^{T}(t) \mathscr{R}_{v, i} \dot{\zeta}(t)+\sum_{v=1}^{m} \alpha_{2, v} \dot{\zeta}^{T}(t) \mathscr{R}_{v} \dot{\zeta}(t)-\sum_{v=1}^{m} \int_{t-d_{v}}^{t} \dot{\zeta}^{T}(s) \mathscr{R}_{v, i} \dot{\zeta}(s) d s\right] \\
& +\sum_{v=1}^{m} \int_{-d_{v}}^{0} \int_{t+\theta}^{t} \mathrm{e}^{2 \alpha(s-\theta)} \dot{\zeta}^{T}(s)\left(\sum_{j=1}^{N} r_{i j} \mathscr{R}_{v, j}\right) \dot{\zeta}(s) d s d \theta \\
& -\mathrm{e}^{2 \alpha t} \sum_{v=1}^{m} \int_{-d_{v}}^{0} \int_{t+\theta}^{t} \dot{\zeta}^{T}(s) \mathscr{R}_{v} \dot{\zeta}(s) d s d \theta .
\end{aligned}
$$

Similarly, the inequality (12) implies the following inequality:

$$
\sum_{v=1}^{m} \int_{-d_{v}}^{0} \int_{t+\theta}^{t} \mathrm{e}^{2 \alpha(s-\theta)} \dot{\zeta}^{T}(s)\left(\sum_{j=1}^{N} r_{i j} \mathscr{R}_{v, j}\right) \dot{\zeta}(s) d s d \theta-\mathrm{e}^{2 \alpha t} \sum_{v=1}^{m} \int_{-d_{v}}^{0} \int_{t+\theta}^{t} \dot{\zeta}^{T}(s) \mathscr{R}_{v} \dot{\zeta}(s) d s d \theta \leq 0 .
$$

We further apply Lemma 2 and get

$$
\begin{aligned}
-\sum_{v=1}^{m} \int_{t-d_{v}}^{t} \dot{\zeta}^{T}(s) \mathscr{R}_{v, i} \dot{\zeta}(s) d s \leq & \sum_{v=1}^{m} \zeta^{T}(t)\left[d_{v} \sum_{\kappa=1}^{3} \frac{1}{2 \kappa-1} \pi_{12, v}^{T} M_{\kappa, v, i} \mathscr{R}_{v, i}^{-1} M_{\kappa, v, i}^{T} \pi_{12, v}\right. \\
& \left.+\sum_{v=1}^{m} \operatorname{sym}\left\{\pi_{12, v}^{T}\left(M_{1, v, i} \pi_{7, v}+M_{2, v, i} \pi_{8, v}+M_{3, v, i} \pi_{9, v}\right)\right\}\right] \zeta(t),
\end{aligned}
$$

that is,

$$
\begin{aligned}
& \mathcal{L} \mathcal{V}_{3}(\varsigma(t), i, t) \leq \mathrm{e}^{2 \alpha t}\left[\sum_{v=1}^{m} \alpha_{1, v} \dot{\zeta}^{T}(t) \mathscr{R}_{v, i} \dot{\zeta}(t)+\sum_{v=1}^{m} \alpha_{2, v} \dot{\varsigma}^{T}(t) \mathscr{R}_{v} \dot{\varsigma}(t)-\sum_{v=1}^{m} \int_{t-d_{v}}^{t} \dot{\varsigma}^{T}(s) \mathscr{R}_{v, i} \dot{\zeta}(s) d s\right] \\
& \leq \mathrm{e}^{2 \alpha t} \zeta^{T}(t)\left\{\left[e_{3 m+4}^{T}\left(\sum_{v=1}^{m} \alpha_{1, v} \mathscr{R}_{v, i}+\sum_{v=1}^{m} \alpha_{2, v} \mathscr{R}_{v}\right) e_{3 m+4}\right]\right. \\
& +\left[\sum_{v=1}^{m} d_{v} \sum_{\kappa=1}^{3} \frac{1}{2 \kappa-1} \pi_{12, v}^{T} M_{\kappa, v, i} \mathscr{R}_{v, i}^{-1} M_{\kappa, v, i}^{T} \pi_{12, v}\right. \\
& \left.\left.+\sum_{v=1}^{m} \operatorname{sym}\left\{\pi_{12, v}^{T}\left(M_{1, v, i} \pi_{7, v}+M_{2, v, i} \pi_{8, v}+M_{3, v, i} \pi_{9, v}\right)\right\}\right]\right\} \zeta(t) . \\
& \mathcal{L} \mathcal{V}_{4}(\varsigma(t), i, t)=\mathrm{e}^{2 \alpha t} \dot{\varsigma}^{T}(t)\left(U_{i}+h_{M} U\right) \dot{\zeta}(t)-\int_{t-h_{M}}^{t} \mathrm{e}^{2 \alpha s} \dot{\zeta}^{T}(s) U \dot{\zeta}(s) d s+\int_{t_{k}}^{t} \mathrm{e}^{2 \alpha s} \dot{\zeta}^{T}(s)\left(\sum_{j=1}^{N} r_{i j} U_{j}\right) \dot{\varsigma}(s) d s \\
& \leq \mathrm{e}^{2 \alpha t} \dot{\zeta}^{T}(t)\left(U_{i}+h_{M} U\right) \dot{\zeta}(t)-\int_{t_{k}}^{t} \mathrm{e}^{2 \alpha s} \dot{\zeta}^{T}(s) U \dot{\zeta}(s) d s+\int_{t_{k}}^{t} \mathrm{e}^{2 \alpha s} \dot{\zeta}^{T}(s)\left(\sum_{j=1}^{N} r_{i j} U_{j}\right) \dot{\zeta}(s) d s .
\end{aligned}
$$

The inequality (13) implies the following inequality:

$$
\int_{t_{k}}^{t} \mathrm{e}^{2 \alpha\left(s+t-t_{k}\right)} \dot{\zeta}^{T}(s)\left(\sum_{j=1}^{N} r_{i j} U_{j}\right) \dot{\zeta}(s) d s-\int_{t_{k}}^{t} \mathrm{e}^{2 \alpha\left(s+t-t_{k}\right)} \dot{\zeta}^{T}(s) U \dot{\zeta}(s) d s \leq 0,
$$

so it is easy to get

$$
\begin{aligned}
\mathcal{L V}_{4}(\varsigma(t), i, t) & \leq \mathrm{e}^{2 \alpha t} \dot{\varsigma}^{T}(t)\left(U_{i}+h_{M} U\right) \dot{\zeta}(t) \\
& =\mathrm{e}^{2 \alpha t} \zeta^{T}(t) e_{3 m+4}^{T}\left(U_{i}+h_{M} U\right) e_{3 m+4} \zeta(t) \\
\mathcal{L} \mathcal{V}_{5}(\varsigma(t), i, t) & =\mathrm{e}^{2 \alpha t}\left\{\left[\varsigma^{T}(t)-\varsigma\left(t_{k}\right)^{T}\right]\left(4 \alpha X_{i}+2\left(\sum_{j=1}^{N} r_{i j} X_{j}\right)\right)\left[\varsigma(t)-\varsigma\left(t_{k+1}\right)\right]\right.
\end{aligned}
$$




$$
\begin{aligned}
& \left.+2 \dot{\varsigma}^{T}(t) X_{i}\left[\varsigma(t)-\varsigma\left(t_{k}+1\right)\right]+2\left[\varsigma^{T}(t)-\varsigma\left(t_{k}\right)^{T}\right] X_{i} \dot{\zeta}(t)\right\} \\
= & 2 \mathrm{e}^{2 \alpha t} \zeta^{T}(t)\left[\pi_{14}^{T}\left(2 \alpha X_{i}+\left(\sum_{j=1}^{N} r_{i j} X_{j}\right)\right) \pi_{15}+e_{3 m+4}^{T} X_{i} \pi_{15}+\pi_{14}^{T} X_{i} e_{3 m+4}\right] \zeta(t) . \\
\mathcal{L} \mathcal{V}_{6}(\varsigma(t), i, t)= & \mathrm{e}^{2 \alpha t} \zeta^{T}(t) \pi_{13}^{T}\left\{2 \alpha\left(t_{k+1}-t\right)\left(t-t_{k}\right) Z_{i}+\left(t_{k+1}-t\right) Z_{i}-\left(t-t_{k}\right) Z_{i}\right.
\end{aligned}
$$$$
\left.+\left(t_{k+1}-t\right)\left(t-t_{k}\right)\left(\sum_{j=1}^{N} r_{i j} Z_{j}\right)\right\} \pi_{13} \zeta(t)
$$$$
\leq \mathrm{e}^{2 \alpha t} \zeta^{T}(t) \pi_{13}^{T}\left\{2 \alpha h_{M}^{2} Z_{i}+\left[\left(t_{k+1}-t\right)-\left(t-t_{k}\right)\right] Z_{i}+h_{M}^{2}\left(\sum_{j=1}^{N} r_{i j} Z_{j}\right)\right\} \pi_{13} \zeta(t) .
$$

$\mathcal{L} \mathcal{V}_{7}(\varsigma(t), i, t)=2 \mathrm{e}^{2 \alpha t} \zeta^{T}(t)\left\{\pi_{1}^{T}\left(Y_{i} \pi_{2}+S_{i} \pi_{3}\right)+\left(t_{k+1}-t\right)\left[\pi_{5}^{T}\left(Y_{i} \pi_{2}+S_{i} \pi_{3}\right)+\pi_{10}^{T} Y_{i} \pi_{4}\right]\right.$

$$
\begin{aligned}
& \left.+\left(t-t_{k}\right)\left[\pi_{6}^{T}\left(Y_{i} \pi_{2}+S_{i} \pi_{3}\right)+\pi_{11}^{T} Y_{i} \pi_{4}\right]\right\} \zeta(t) \\
& +4 \alpha \mathrm{e}^{2 \alpha t} \zeta^{T}(t)\left\{\left(t_{k+1}-t\right)\left[\pi_{10}^{T}\left(Y_{i} \pi_{2}+S_{i} \pi_{3}\right)\right]+\left(t-t_{k}\right)\left[\pi_{11}^{T}\left(Y_{i} \pi_{2}+S_{i} \pi_{3}\right)\right]\right\} \zeta(t) \\
& +2 \mathrm{e}^{2 \alpha t} \zeta^{T}(t)\left\{\left(t_{k+1}-t\right)\left[\pi_{10}^{T}\left(\left(\sum_{j=1}^{N} r_{i j} Y_{j}\right) \pi_{2}+\left(\sum_{j=1}^{N} r_{i j} S_{j}\right) \pi_{3}\right)\right]\right. \\
& \left.+\left(t-t_{k}\right)\left[\pi_{11}^{T}\left(\left(\sum_{j=1}^{N} r_{i j} Y_{j}\right) \pi_{2}+\left(\sum_{j=1}^{N} r_{i j} S_{j}\right) \pi_{3}\right)\right]\right\} \zeta(t) \\
& =2 \mathrm{e}^{2 \alpha t} \zeta^{T}(t)\left\{\pi_{1}^{T}\left(Y_{i} \pi_{2}+S_{i} \pi_{3}\right)+\left(t_{k+1}-t\right)\left[\left(\pi_{5}^{T}+2 \alpha \pi_{10}^{T}\right)\left(Y_{i} \pi_{2}+S_{i} \pi_{3}\right)+\pi_{10}^{T} Y_{i} \pi_{4}\right.\right. \\
& \left.+\pi_{10}^{T}\left(\left(\sum_{j=1}^{N} r_{i j} Y_{j}\right) \pi_{2}+\left(\sum_{j=1}^{N} r_{i j} S_{j}\right) \pi_{3}\right)\right]+\left(t-t_{k}\right)\left[\left(\pi_{6}^{T}+2 \alpha \pi_{11}^{T}\right)\left(Y_{i} \pi_{2}+S_{i} \pi_{3}\right)\right. \\
& \left.\left.+\pi_{11}^{T} Y_{i} \pi_{4}+\pi_{11}^{T}\left(\left(\sum_{j=1}^{N} r_{i j} Y_{j}\right) \pi_{2}+\left(\sum_{j=1}^{N} r_{i j} S_{j}\right) \pi_{3}\right)\right]\right\} \zeta(t) .
\end{aligned}
$$

In addition, for any a free weighting matrix $G_{i}$, and scalars $\gamma$ and $\delta$, we always have

$$
\begin{aligned}
0= & 2 \mathrm{e}^{2 \alpha t}\left[\delta \varsigma^{T}(t) G_{i}+\dot{\zeta}^{T}(t) G_{i}+\gamma \varsigma^{T}\left(t_{k}\right) G_{i}\right] \\
& \times\left[-\dot{\zeta}(t)+\mathscr{A}_{i} \varsigma(t)+\sum_{v=1}^{m} \mathscr{B}_{v, i} \varsigma\left(t-d_{v}\right)+\mathscr{W}_{i} \xi(\mathscr{D} \varsigma(t), y(t))-\mathscr{K}_{i} \mathscr{C}_{i} \varsigma\left(t_{k}\right)\right] \\
= & 2 \mathrm{e}^{2 \alpha t} \zeta^{T}(t)\left[\left(\delta e_{1}^{T} G_{i}+e_{3 m+4}^{T} G_{i}+\gamma e_{3 m+2}^{T} G_{i}\right)\right. \\
& \left.\times\left(-e_{3 m+4}+\mathscr{A}_{i} e_{1}+\sum_{v=1}^{m} \mathscr{B}_{v, i} e_{v+1}+\mathscr{W}_{i} e_{3 m+5}-\mathscr{K}_{i} \mathscr{C}_{i} e_{3 m+2}\right)\right] \zeta(t) .
\end{aligned}
$$

Furthermore, on the basis of (5), the following inequality is satisfied:

$$
\begin{aligned}
0 \leq & 2 \mathrm{e}^{2 \alpha t} \frac{t_{k+1}-t}{h_{k}}\left[\varsigma(t)^{T} \mathscr{D}^{T} \mathscr{S} \mathscr{V}_{1, i} \xi(\mathscr{D} \varsigma(t), y(t))-\xi(\mathscr{D} \varsigma(t), y(t))^{T} \mathscr{V}_{1, i} \xi(\mathscr{D} \varsigma(t), y(t))\right] \\
& +2 \mathrm{e}^{2 \alpha t} \frac{t-t_{k}}{h_{k}}\left[\varsigma(t)^{T} \mathscr{D}^{T} \mathscr{S} \mathscr{V}_{2, i} \xi(\mathscr{D} \varsigma(t), y(t))-\xi(\mathscr{D} \varsigma(t), y(t))^{T} \mathscr{V}_{2, i} \xi(\mathscr{D} \varsigma(t), y(t))\right], \\
= & 2 \mathrm{e}^{2 \alpha t} \zeta^{T}(t)\left[\frac{t_{k+1}-t}{h_{k}}\left(e_{1}^{T} \mathscr{D}^{T} \mathscr{S}_{1, i} e_{3 m+5}-e_{3 m+5}^{T} \mathscr{V}_{1, i} e_{3 m+5}\right)\right. \\
& \left.+\frac{t-t_{k}}{h_{k}}\left(e_{1}^{T} \mathscr{D}^{T} \mathscr{S} \mathscr{V}_{2, i} e_{3 m+5}-e_{3 m+5}^{T} \mathscr{V}_{2, i} e_{3 m+5}\right)\right] \zeta(t) .
\end{aligned}
$$


We insert the right side of (19) and (20) to $\mathcal{L V}$ and let $L_{i}=G_{i} \mathscr{K}_{i}$, then $\mathcal{L} \mathcal{V}(\varsigma(t), i, t)$ can be expressed as:

$$
\mathcal{L} \mathcal{V}(\varsigma(t), i, t) \leq \mathrm{e}^{2 \alpha t} \zeta^{T}(t)\left[\frac{t_{k+1}-t}{h_{k}} \hat{\Xi}_{1}\left(h_{k}\right)+\frac{t-t_{k}}{h_{k}} \hat{\Xi}_{2}\left(h_{k}\right)\right] \zeta(t),
$$

where

$$
\begin{aligned}
& \hat{\Xi}_{1}\left(h_{k}\right)=\Phi_{1}+\Phi_{2}+h_{k} \Phi_{3}+\sum_{v=1}^{m} \sum_{\kappa=1}^{3} \frac{d_{v}}{2 \kappa-1} \pi_{12, v}^{T} M_{\kappa, v, i} \mathscr{R}_{v, i}^{-1} M_{\kappa, v, i}^{T} \pi_{12, v}, \\
& \hat{\Xi}_{2}\left(h_{k}\right)=\Phi_{1}+\Phi_{4}+h_{k} \Phi_{5}+\sum_{v=1}^{m} \sum_{\kappa=1}^{3} \frac{d_{v}}{2 \kappa-1} \pi_{12, v}^{T} M_{\kappa, v, i} \mathscr{R}_{v, i}^{-1} M_{\kappa, v, i}^{T} \pi_{12, v} .
\end{aligned}
$$

It is noted that

$$
\begin{aligned}
& \hat{\Xi}_{1}\left(h_{k}\right)=\frac{h_{k}}{h_{M}} \hat{\Xi}_{1}\left(h_{M}\right)+\frac{h_{M}-h_{k}}{h_{M}} \hat{\Xi}_{1}\left(h_{m}\right), \\
& \hat{\Xi}_{2}\left(h_{k}\right)=\frac{h_{k}}{h_{M}} \hat{\Xi}_{2}\left(h_{M}\right)+\frac{h_{M}-h_{k}}{h_{M}} \hat{\Xi}_{2}\left(h_{m}\right) .
\end{aligned}
$$

From (9) and (13), we can find that

$$
\hat{\Xi}_{1}\left(h_{k}\right)<0, \quad \hat{\Xi}_{2}\left(h_{k}\right)<0
$$

Thus, we can further get that

$$
\mathcal{L} \mathcal{V}(\varsigma(t), i, t) \leq 0, \quad t \in\left[t_{k}, t_{k+1}\right)
$$

It suggests that, for $t \in\left[t_{k}, t_{k+1}\right)$, one has

$$
\mathbb{E}\{\mathcal{V}(t)\} \leq \mathbb{E}\left\{\mathcal{V}\left(t_{k}\right)\right\} \leq \mathbb{E}\left\{\mathcal{V}\left(t_{k-1}\right)\right\} \leq \ldots \leq \mathbb{E}\{\mathcal{V}(0)\}
$$

On the basis of Lemma 1 and (23), we can conclude that

$$
\begin{aligned}
\mathbb{E}\left\{\|\varsigma(t)\|^{2}\right\} \leq & \theta_{1} \mathbb{E}\left\{\left\|\varsigma\left(t_{k}\right)\right\|^{2}\right\}+\mathbb{E}\left\{\sum_{v=1}^{m} \int_{t_{k}-d_{v}}^{t_{k}} \theta_{2} \varepsilon\|\varsigma(s)\|^{2} d s\right\} \\
= & \sum_{v=1}^{m} \frac{\theta_{1}}{\lambda_{\min }\left(P_{i}\right) \mathrm{e}^{2 \alpha t_{k}}} \mathrm{e}^{2 \alpha t_{k}} \lambda_{\min }\left(P_{i}\right) \mathbb{E}\left\{\left\|\varsigma\left(t_{k}\right)\right\|^{2}\right\} \\
& +\mathbb{E}\left\{\sum_{v=1}^{m} \int_{t_{k}-d_{v}}^{t_{k}} \frac{\theta_{2} \lambda_{\min }\left(Q_{v, i}\right) \mathrm{e}^{2 \alpha\left(t_{k}+d_{v}\right)} \varepsilon\|\varsigma(s)\|^{2} d s}{\lambda_{\min }\left(Q_{v, i}\right) \mathrm{e}^{2 \alpha\left(t_{k}+d_{v}\right)}}\right\} \\
\leq & \sum_{v=1}^{m} \frac{\theta_{1}}{\lambda_{\min }\left(P_{i}\right) \mathrm{e}^{2 \alpha t_{k}}} \mathbb{E}\left\{\mathrm{e}^{2 \alpha t_{k}} \varsigma^{T}\left(t_{k}\right) P_{i} z\left(t_{k}\right)\right\} \\
& +\mathbb{E}\left\{\sum_{v=1}^{m} \frac{\theta_{2} \varepsilon \int_{t_{k}-d_{v}}^{t_{k}} \mathrm{e}^{2 \alpha\left(t_{k}+d_{v}\right)} \varsigma^{T}(s)\left(Q_{v, i}\right) \varsigma(s) d s}{\lambda_{\min }\left(Q_{v, i}\right) \mathrm{e}^{2 \alpha\left(t_{k}+d_{v}\right)}}\right\} \\
\leq & \sum_{v=1}^{m} \max \left\{\frac{\theta_{1}}{\lambda_{\min }\left(P_{i}\right) \mathrm{e}^{2 \alpha t_{k}}}, \frac{\theta_{2} \varepsilon}{\lambda_{\min }\left(Q_{v, i}\right) \mathrm{e}^{2 \alpha\left(t_{k}+d_{v}\right)}}\right\} \mathbb{E}\left\{\mathcal{V}_{1}\left(t_{k}\right)+\mathcal{V}_{2}\left(t_{k}\right)\right\} \\
\leq & \sum_{v=1}^{m} \max \left\{\frac{\theta_{1}}{\lambda_{\min }\left(P_{i}\right) \mathrm{e}^{2 \alpha t_{k}}}, \frac{\theta_{2} \varepsilon}{\lambda_{\min }\left(Q_{v, i}\right) \mathrm{e}^{2 \alpha\left(t_{k}+d_{v}\right)}}\right\} \mathbb{E}\left\{\mathcal{V}\left(t_{k}\right)\right\}
\end{aligned}
$$




$$
\leq \sum_{v=1}^{m} \mathrm{e}^{2 \alpha h_{M}} \max \left\{\frac{\theta_{1}}{\lambda_{\min }\left(P_{i}\right)}, \frac{\theta_{2} \varepsilon}{\lambda_{\min }\left(Q_{v, i}\right) \mathrm{e}^{2 \alpha d_{v}}}\right\} \mathrm{e}^{-2 \alpha t} \mathbb{E}\{\mathcal{V}(0)\}
$$

It can be computed that

$$
\begin{aligned}
& \mathbb{E}\{\mathcal{V}(0)\}=\mathbb{E}\left\{\varsigma^{T}(0) P_{i} \varsigma(0)\right\}+\mathbb{E}\left\{\sum_{v=1}^{m} \int_{-d_{v}}^{0} \mathrm{e}^{2 \alpha\left(s+d_{v}\right)} \varsigma^{T}(s) Q_{v, i} \varsigma(s) d s\right\} \\
& +\mathbb{E}\left\{\sum_{v=1}^{m} \int_{-d_{v}}^{0} \int_{\theta}^{0} \mathrm{e}^{2 \alpha(s-\theta)} \varsigma^{T}(s) Q_{v} \varsigma(s) d s d \theta\right\} \\
& +\mathbb{E}\left\{\sum_{v=1}^{m} \int_{-d_{v}}^{0} \int_{\theta}^{0} \mathrm{e}^{2 \alpha(s-\theta)} \dot{\zeta}^{T}(s) \mathscr{R}_{v, i} \dot{\zeta}(s) d s d \theta\right\} \\
& +\mathbb{E}\left\{\sum_{v=1}^{m} \int_{-d_{v}}^{0} \int_{\theta}^{0} \int_{\beta}^{0} \mathrm{e}^{2 \alpha(s-\beta)} \dot{\varsigma}^{T}(s) \mathscr{R}_{v} \dot{\zeta}(s) d s d \theta\right\} \\
& +\mathbb{E}\left\{\int_{-h_{M}}^{0} \int_{\theta}^{0} \mathrm{e}^{2 \alpha s} \dot{\zeta}^{T}(s) U \dot{\zeta}(s) d s d \theta\right\} \\
& \leq \mathbb{E}\left\{\lambda_{\max }\left(P_{i}\right)\|\varsigma(0)\|^{2}\right\}+\mathbb{E}\left\{\sum_{v=1}^{m} \alpha_{1, v} \lambda_{\max }\left(Q_{v, i}\right) \sup _{-d_{v} \leq \theta \leq 0}\|\varsigma(\theta)\|^{2}\right\} \\
& +\mathbb{E}\left\{\sum_{v=1}^{m} \alpha_{2, v} \lambda_{\max }\left(Q_{v}\right) \sup _{-d_{v} \leq \theta \leq 0}\|\varsigma(\theta)\|^{2}\right\}+\mathbb{E}\left\{\sum_{v=1}^{m} \alpha_{2, v} \lambda_{\max }\left(\mathscr{R}_{v, i}\right) \sup _{-d_{v} \leq \theta \leq 0}\|\dot{\varsigma}(\theta)\|^{2}\right\} \\
& +\mathbb{E}\left\{\sum_{v=1}^{m} \alpha_{3, v} \lambda_{\max }\left(\mathscr{R}_{v}\right) \sup _{-d_{v} \leq \theta \leq 0}\|\dot{\zeta}(\theta)\|^{2}\right\}+\mathbb{E}\left\{\alpha_{4} \lambda_{\max }(U) \sup _{-h_{M} \leq \theta \leq 0}\|\dot{\zeta}(\theta)\|^{2}\right\} \\
& \leq\left(\varrho_{1}+\varrho_{2}+\varrho_{3}\right) \sup _{-d_{v} \leq \theta \leq 0} \mathbb{E}\left\{\|\varsigma(\theta)\|^{2}\right\}+\left(\varrho_{4}+\varrho_{5}+\varrho_{6}\right) \sup _{-\chi \leq \theta \leq 0} \mathbb{E}\left\{\|\dot{\zeta}(\theta)\|^{2}\right\} \\
& \leq \varrho \sup _{-\chi \leq \theta \leq 0} \mathbb{E}\left\{\|\varsigma(\theta)\|^{2}+\|\dot{\zeta}(\theta)\|^{2}\right\},
\end{aligned}
$$

where $\varrho=\max \left\{\varrho_{1}+\varrho_{2}+\varrho_{3}, \varrho_{4}+\varrho_{5}+\varrho_{6}\right\}$,

$$
\begin{aligned}
\varrho_{1} & =\max _{i \in \mathcal{N}} \lambda_{\max }\left(P_{i}\right), \\
\varrho_{2} & =\sum_{v=1}^{m} \alpha_{1, v} \max _{i \in \mathcal{N}} \lambda_{\max }\left(Q_{v, i}\right), \\
\varrho_{3} & =\sum_{v=1}^{m} \alpha_{2, v} \lambda_{\max }\left(Q_{v}\right), \\
\varrho_{4} & =\sum_{v=1}^{m} \alpha_{2, v} \max _{i \in \mathcal{N}} \lambda_{\max }\left(\mathscr{R}_{v, i}\right), \\
\varrho_{5} & =\sum_{v=1}^{m} \alpha_{3, v} \lambda_{\max }\left(\mathscr{R}_{v}\right), \\
\varrho_{6} & =\alpha_{4} \lambda_{\max }(U), \\
\chi & =\max \left\{d_{v}, h_{M}\right\}, v=1,2, \ldots, m .
\end{aligned}
$$

Combining (24) with (25), we can get that

$$
\mathbb{E}\left\{\|\varsigma(t)\|^{2}\right\} \leq \sum_{v=1}^{m} \mathrm{e}^{2 \alpha h_{M}} \max \left\{\frac{\theta_{1}}{\lambda_{\min }\left(P_{i}\right)}, \frac{\theta_{2} \varepsilon}{\lambda_{\min }\left(Q_{v, i}\right) \mathrm{e}^{2 \alpha d_{v}}}\right\} \varrho \mathrm{e}^{-2 \alpha t} \mathbb{E}\left\{\left\|\varsigma_{0}\right\|_{c}^{2}\right\}, v=1,2, \ldots, m .
$$

Hence, by Definition 1, the master-slave systems (1) and (2) are exponentially synchronous in the mean square.

Remark 2. In the proof of Theorem 1, we introduced mode-dependent single/double integral terms and considered the mode-dependent sampled-state information to construct functionals. For example, since dif- 
ferent $U_{i}$ is selected for distinct system mode, $\int_{t_{k}}^{t} e^{2 \alpha s} \dot{\zeta}^{T}(s)\left(\sum_{j=1}^{N} r_{i j} U_{j}\right) \dot{\zeta}(s) d s$ is consequentially involved in $\mathcal{L V}_{4}(\varsigma(t), i, t)$. To achieve the stability result, we need to cope with it. As seen clearly from (18), $\int_{t_{k}}^{t} e^{2 \alpha s} \dot{\zeta}^{T}(s)\left(\sum_{j=1}^{N} r_{i j} U_{j}\right) \dot{\zeta}(s) d s$ is efficiently eliminated by $-\int_{-h_{M}}^{0} \int_{t+\theta}^{t} e^{2 \alpha s} \dot{\zeta}^{T}(s) U \dot{\varsigma}(s) d s d \theta$.

Similarly, it can be seen from (16) and (17), the introduction of $\sum_{v=1}^{m} \int_{-d_{v}}^{0} \int_{t+\theta}^{t} e^{2 \alpha(s-\theta)} \varsigma^{T}(s) Q_{v} \varsigma(s) d s d \theta$ and $\sum_{v=1}^{m} \int_{-d_{v}}^{0} \int_{\theta}^{0} \int_{t+\beta}^{t} e^{2 \alpha(s-\beta)} \dot{\zeta}^{T}(s) \mathscr{R}_{v} \dot{\zeta}(s) d s d \theta$ is to ensure distinct $Q_{v, i}$ and $\mathscr{R}_{v, i}$ can be applied to different system mode, that's the reason why the mode-independent double/triple integral terms are constructed in the Lyapunov functionals.

Remark 3. In Theorem 1, one of the innovation of Lyapunov functionals $\mathcal{V}(\varsigma(t), i, t)$ in (15) lies in that some mode-dependent two-sided functions i.e., $\mathcal{V}_{5}, \mathcal{V}_{6}, \mathcal{V}_{7}$ which not only fully utilize the interval message from $\varsigma\left(t_{k}\right)$ to $\varsigma(t)$ but also consider the information from $\varsigma(t)$ to $\varsigma\left(t_{k+1}\right)$. The looped-functional approach has been used to analyze system stability in [42]. However, Ref. [42] did not consider the influence of Markovian parameters on the construction of functionals. To bridge this gap, this paper constructs mode-dependent looped-functional.

To make comparison, on the basis of Theorem 1, a simplified delayed system is proposed in which Markovian parameters is not considered.

Consider the following master system:

$$
\mathscr{M}:\left\{\begin{array}{l}
\dot{x}(t)=\mathscr{A} x(t)+\mathscr{B} x(t-d)+\mathscr{W} \eta(\mathscr{D} x(t)), \\
q(t)=\mathscr{C} x(t)
\end{array}\right.
$$

and slave system

$$
\mathscr{N}:\left\{\begin{array}{l}
\dot{y}(t)=\mathscr{A} y(t)+\mathscr{B} y(t-d)+\mathscr{W} \eta(\mathscr{D} y(t))+u(t) \\
p(t)=\mathscr{C} y(t)
\end{array}\right.
$$

where $u(t)=\mathscr{K}\left(q\left(t_{k}\right)-p\left(t_{k}\right)\right), t_{k} \leq t<t_{k+1}, d$ is the constant time-delay. Hence, the error system (3) reduces to

$$
\dot{\varsigma}(t)=\mathscr{A} \varsigma(t)+\mathscr{B} \varsigma(t-d)+\mathscr{W} \xi(\mathscr{D} \varsigma(t), y(t))-\mathscr{K} \mathscr{C} \varsigma\left(t_{k}\right)
$$

For simplicity, we use nomenclature for vectors and matrices in the following.

$$
\begin{aligned}
& \omega_{3}=\frac{1}{d} \int_{t-d}^{t} \varsigma(s) d s, \quad \omega_{4}=\frac{2}{d^{2}} \int_{t-d}^{t} \int_{t-d}^{s} \varsigma(u) d u d s, \quad \alpha_{5}=\frac{\mathrm{e}^{2 \alpha d}-1}{2 \alpha}, \\
& \bar{\zeta}^{T}(t)=\left[\varsigma^{T}(t), \quad \varsigma^{T}(t-d), \quad \omega_{3}^{T}(t), \quad \omega_{4}^{T}(t), \quad \varsigma^{T}\left(t_{k}\right), \varsigma^{T}\left(t_{k+1}\right), \quad \dot{\varsigma}^{T}(t), \quad \xi^{T}(\mathscr{D} \varsigma(t), y(t))\right], \\
& \bar{e}_{\bar{\iota}}=\left[0_{n \times(\bar{\iota}-1) n}, \quad I_{n \times n}, \quad 0_{n \times(8-\bar{\iota}) n}\right], \quad \bar{\iota}=1,2, \ldots, 8 .
\end{aligned}
$$

Corollary 1. Given scalars $\alpha>0, \delta$, and $\gamma$, the error system (29) is exponentially stable if there exist positive definite matrices $P, Q, \mathscr{R}, U$, matrices $Z, X, Y, S, M_{1}, M_{2}, M_{3}, G, L$, and diagonal matrices 
$\mathscr{V}_{1}>0, \mathscr{V}_{2}>0$ such that the following conditions hold for $h_{k} \in\left\{h_{m}, h_{M}\right\}$ :

$$
\begin{aligned}
& \check{\Xi}_{1}\left(h_{k}\right)=\left[\begin{array}{cc}
\bar{\Phi}_{1}+\bar{\Phi}_{2}+h_{k} \bar{\Phi}_{3} & \sqrt{d} \bar{\Xi} \\
* & \overline{\mathscr{R}}
\end{array}\right]<0, \\
& \check{\Xi}_{2}\left(h_{k}\right)=\left[\begin{array}{cc}
\bar{\Phi}_{1}+\bar{\Phi}_{4}+h_{k} \bar{\Phi}_{5} & \sqrt{d} \bar{\Xi} \\
* & \overline{\mathscr{R}}
\end{array}\right]<0,
\end{aligned}
$$

where

$$
\begin{aligned}
& \bar{\pi}_{1}=\left[\begin{array}{ll}
\bar{e}_{5}^{T}-\bar{e}_{1}^{T}, & \bar{e}_{1}^{T}-\bar{e}_{6}^{T}
\end{array}\right]^{T}, \quad \bar{\pi}_{2}=\left[\begin{array}{ll}
\bar{e}_{1}^{T}-\bar{e}_{5}^{T}, & \bar{e}_{1}^{T}-\bar{e}_{6}^{T}
\end{array}\right]^{T}, \quad \bar{\pi}_{3}=\left[\begin{array}{ll}
\bar{e}_{5}^{T}, & \bar{e}_{6}^{T}
\end{array}\right]^{T}, \\
& \bar{\pi}_{4}=\left[\begin{array}{ll}
\bar{e}_{7}^{T}, & \bar{e}_{7}^{T}
\end{array}\right]^{T}, \quad \bar{\pi}_{5}=\left[\begin{array}{ll}
\bar{e}_{7}^{T}, & 0
\end{array}\right]^{T}, \quad \bar{\pi}_{6}=\left[\begin{array}{ll}
0, & \bar{e}_{7}^{T}
\end{array}\right]^{T}, \quad \bar{\pi}_{7}=\bar{e}_{1}-\bar{e}_{2}, \\
& \bar{\pi}_{8}=\bar{e}_{1}+\bar{e}_{2}-2 \bar{e}_{3}, \quad \bar{\pi}_{9}=\bar{e}_{1}-\bar{e}_{2}-6 \bar{e}_{3}+6 \bar{e}_{4}, \quad \bar{\pi}_{10}=\left[\begin{array}{ll}
\bar{e}_{1}^{T}-\bar{e}_{5}^{T}, & 0
\end{array}\right]^{T},
\end{aligned}
$$

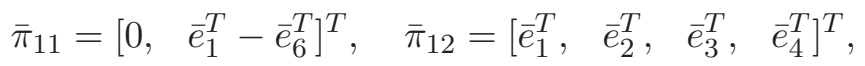

$$
\begin{aligned}
& \bar{\pi}_{13}=\left[\begin{array}{ll}
\bar{e}_{5}^{T}, & \bar{e}_{6}^{T}
\end{array}\right]^{T}, \quad \bar{\pi}_{14}=\bar{e}_{1}-\bar{e}_{5}, \quad \bar{\pi}_{15}=\bar{e}_{1}-\bar{e}_{6}, \\
& \bar{\Phi}_{1}=\bar{e}_{1}^{T}\left(2 \alpha P+e^{2 \alpha d} Q\right) \bar{e}_{1}-\bar{e}_{2}^{T} Q \bar{e}_{2}+\alpha_{5} \bar{e}_{7}^{T} \mathscr{R} \bar{e}_{7}+\bar{e}_{7}^{T} U \bar{e}_{7}+\bar{\pi}_{13}^{T} 2 \alpha h_{M}^{2} Z \bar{\pi}_{13} \\
& +\operatorname{sym}\left\{\bar{e}_{1}^{T} P \bar{e}_{7}+\bar{\pi}_{1}^{T}\left(Y \bar{\pi}_{2}+S \bar{\pi}_{3}\right)+\bar{\pi}_{14}^{T} 2 \alpha X \bar{\pi}_{15}+\bar{e}_{7}^{T} X \bar{\pi}_{15}+\bar{\pi}_{14}^{T} X \bar{e}_{7}\right. \\
& +\left(\delta \bar{e}_{1}^{T} G+\bar{e}_{7}^{T} G+\gamma \bar{e}_{5}^{T} G\right)\left(-\bar{e}_{7}+\mathscr{A} \bar{e}_{1}+\mathscr{B} \bar{e}_{2}+\mathscr{W} \bar{e}_{8}-\mathscr{K} \mathscr{C} \bar{e}_{5}\right) \\
& \left.+\bar{\pi}_{12}^{T}\left(M_{1} \bar{\pi}_{7}+M_{2} \bar{\pi}_{8}+M_{3} \bar{\pi}_{9}\right)\right\}, \\
& \bar{\Phi}_{2}=\operatorname{sym}\left\{\bar{e}_{1}^{T} \mathscr{D}^{T} \mathscr{S} \bar{V}_{1} \bar{e}_{8}\right\}-2 \bar{e}_{8}^{T} \mathscr{V}_{1} \bar{e}_{8}, \\
& \bar{\Phi}_{3}=\operatorname{sym}\left\{\left(\bar{\pi}_{5}^{T}+2 \alpha \bar{\pi}_{10}^{T}\right)\left(Y \bar{\pi}_{2}+S \bar{\pi}_{3}\right)+\bar{\pi}_{10}^{T} Y \bar{\pi}_{4}\right\}+\bar{\pi}_{13}^{T} Z \bar{\pi}_{13}^{T}, \\
& \bar{\Phi}_{4}=\operatorname{sym}\left\{\bar{e}_{1}^{T} \mathscr{D}^{T} \mathscr{S}_{V_{2}} \bar{e}_{8}\right\}-2 \bar{e}_{8}^{T} \mathscr{V}_{2} \bar{e}_{8}, \\
& \bar{\Phi}_{5}=\operatorname{sym}\left\{\left(\bar{\pi}_{6}^{T}+2 \alpha \bar{\pi}_{11}^{T}\right)\left(Y \bar{\pi}_{2}+S \bar{\pi}_{3}\right)+\bar{\pi}_{11}^{T} Y \bar{\pi}_{4}\right\}-\bar{\pi}_{13}^{T} Z \bar{\pi}_{13}, \\
& \Xi=\left[\begin{array}{lll}
\bar{\pi}_{12}^{T} M_{1} & \bar{\pi}_{12}^{T} M_{2} & \bar{\pi}_{12}^{T} M_{3}
\end{array}\right], \\
& \overline{\mathscr{R}}=\operatorname{diag}\left\{\begin{array}{lll}
-\mathscr{R} & -3 \mathscr{R} & -5 \mathscr{R}
\end{array}\right\} .
\end{aligned}
$$

Proof. Introduce simplified two-sided looped-functional,

$$
V(t)=\sum_{\epsilon=1}^{7} V_{\epsilon}(t)
$$

with

$$
\begin{aligned}
& V_{1}(t)=\mathrm{e}^{2 \alpha t} \varsigma^{T}(t) P \varsigma(t), \\
& V_{2}(t)=\int_{t-d}^{t} \mathrm{e}^{2 \alpha(s+d)} \varsigma^{T}(s) Q \varsigma(s) d s, \\
& V_{3}(t)=\int_{-d}^{0} \int_{t+\theta}^{t} \mathrm{e}^{2 \alpha(s-\theta)} \dot{\varsigma}^{T}(s) \mathscr{R} \dot{\zeta}(s) d s d \theta, \\
& V_{4}(t)=\int_{t_{k}}^{t} \mathrm{e}^{2 \alpha s} \dot{\varsigma}^{T}(s) U \dot{\varsigma}(s) d s,
\end{aligned}
$$




$$
\begin{aligned}
V_{5}(t) & =2 \mathrm{e}^{2 \alpha t} \mu_{1}^{T}(t) X \mu_{2}(t), \\
V_{6}(t) & =\left(t_{k+1}-t\right)\left(t-t_{k}\right) \mathrm{e}^{2 \alpha t} \zeta_{3}^{T} Z \zeta_{3}, \\
V_{7}(t) & =2 \mathrm{e}^{2 \alpha t} \zeta_{1}^{T}(t)\left[Y \zeta_{2}(t)+S \zeta_{3}\right] .
\end{aligned}
$$

According to the derivative of $V(t)$ along the trajectory of the error system (29), we can obtain

$$
\begin{aligned}
\dot{V}_{1}(t)= & 2 \mathrm{e}^{2 \alpha t} \bar{\zeta}^{T}(t)\left(\bar{e}_{1}^{T} \alpha P \bar{e}_{1}+\bar{e}_{1}^{T} P \bar{e}_{7}\right) \bar{\zeta}(t), \\
\dot{V}_{2}(t)= & \mathrm{e}^{2 \alpha t} \bar{\zeta}^{T}(t)\left(\mathrm{e}^{2 \alpha d} \bar{e}_{1}^{T} Q \bar{e}_{1}-\bar{e}_{2}^{T} Q \bar{e}_{2}\right) \bar{\zeta}(t), \\
\dot{V}_{3}(t)= & \mathrm{e}^{2 \alpha t} \bar{\zeta}^{T}(t)\left\{\operatorname{sym}\left[\bar{\pi}_{12}^{T}\left(M_{1} \bar{\pi}_{7}+M_{2} \bar{\pi}_{8}+M_{3} \bar{\pi}_{9}\right)\right]+\alpha_{5} \bar{e}_{7}^{T} \mathscr{R}_{\bar{e}}\right. \\
& \left.+d \sum_{\kappa=1}^{3} \frac{1}{2 \kappa-1} \bar{\pi}_{12}^{T} M_{\kappa}^{T} \mathscr{R}^{-1} M_{\kappa} \bar{\pi}_{12}\right\} \bar{\zeta}(t), \\
\dot{V}_{4}(t)= & \mathrm{e}^{2 \alpha t} \bar{\zeta}^{T}(t) \bar{e}_{7}^{T} U \bar{e}_{7} \bar{\zeta}(t), \\
\dot{V}_{5}(t)= & 2 \mathrm{e}^{2 \alpha t} \bar{\zeta}^{T}(t)\left(\bar{\pi}_{14}^{T} 2 \alpha X \bar{\pi}_{15}+\bar{e}_{7}^{T} X \bar{\pi}_{15}+\bar{\pi}_{14}^{T} X \bar{e}_{7}\right) \bar{\zeta}(t), \\
\dot{V}_{6}(t)= & \mathrm{e}^{2 \alpha t} \bar{\zeta}^{T}(t) \bar{\pi}_{13}^{T}\left\{2 \alpha h_{M}^{2} Z+\left[\left(t_{k+1}-t\right)-\left(t-t_{k}\right)\right] Z\right\} \bar{\pi}_{13} \bar{\zeta}(t), \\
\dot{V}_{7}(t)= & 2 \mathrm{e}^{2 \alpha t} \bar{\zeta}(t)\left\{\bar{\pi}_{1}^{T}\left(Y \bar{\pi}_{2}+S \bar{\pi}_{3}\right)+\left(t_{k+1}-t\right)\left[\left(\bar{\pi}_{5}^{T}+2 \alpha \bar{\pi}_{10}^{T}\right)\left(Y \bar{\pi}_{2}+S \bar{\pi}_{3}\right)\right.\right. \\
& \left.\left.+\bar{\pi}_{10}^{T} Y \bar{\pi}_{4}\right]+\left(t-t_{k}\right)\left[\left(\bar{\pi}_{6}^{T}+2 \alpha \bar{\pi}_{11}^{T}\right)\left(Y \bar{\pi}_{2}+S \bar{\pi}_{3}\right)+\bar{\pi}_{11}^{T} Y \bar{\pi}_{4}\right]\right\} \bar{\zeta}(t) .
\end{aligned}
$$

In addition, for any free weighting matrix $G$, and scalars $\gamma$ and $\delta$, we always have:

$$
0=2 \mathrm{e}^{2 \alpha t}\left[\delta \varsigma^{T}(t) G+\dot{\zeta}^{T}(t) G+\gamma \varsigma^{T}\left(t_{k}\right) G\right]\left[-\dot{\varsigma}(t)+\mathscr{A} \varsigma(t)+\mathscr{B} \varsigma(t-d)+\mathscr{W} \xi(\mathscr{D} \varsigma(t), y(t))-\mathscr{K} \mathscr{C} \varsigma\left(t_{k}\right)\right],
$$

and similarly the following inequality is satisfied:

$$
\begin{aligned}
0 \leq & 2 \mathrm{e}^{2 \alpha t} \frac{t_{k+1}-t}{h_{k}}\left[\varsigma(t)^{T} \mathscr{D}^{T} \mathscr{S} \mathscr{V}_{1} \xi(\mathscr{D} \varsigma(t), y(t))-\xi(\mathscr{D} \varsigma(t), y(t))^{T} \mathscr{V}_{1} \xi(\mathscr{D} \varsigma(t), y(t))\right] \\
& +2 \mathrm{e}^{2 \alpha t} \frac{t-t_{k}}{h_{k}}\left[\varsigma(t)^{T} \mathscr{D}^{T} \mathscr{S} \mathscr{V}_{2} \xi(\mathscr{D} \varsigma(t), y(t))-\xi(\mathscr{D} \varsigma(t), y(t))^{T} \mathscr{V}_{2} \xi(\mathscr{D} \varsigma(t), y(t))\right] .
\end{aligned}
$$

Furthermore, referring to the proof of Theorem 1, the ASDC gain matrix in (29) is

$$
\mathscr{K}=G^{-1} L
$$

\section{Numerical Examples}

In this section, two examples are given to demonstrate the reduced conservatism of the synchronization criterion and the effectiveness of the ASDC design approach.

Example 1. Consider a two mode $(i \in \mathcal{N}=1,2)$ Chua's circuit with multiple time delays as the master 
system:

$$
\left\{\begin{array}{l}
\dot{x}_{1}(t)=a_{i}\left[x_{2}(t)-m_{i} x_{1}(t)+g\left(x_{1}(t)\right)\right]-c_{1, i} x_{1}\left(t-d_{1}\right)-c_{2, i} x_{1}\left(t-d_{2}\right), \\
\dot{x}_{2}(t)=x_{1}(t)-x_{2}(t)+x_{3}(t)-c_{1, i} x_{1}\left(t-d_{1}\right)-c_{2, i} x_{2}\left(t-d_{2}\right), \\
\dot{x}_{3}(t)=-b_{i} x_{2}(t)+c_{1, i}\left[2 x_{1}\left(t-d_{1}\right)-x_{3}\left(t-d_{1}\right)\right]+c_{2, i}\left[2 x_{1}\left(t-d_{2}\right)-x_{3}\left(t-d_{2}\right)\right] .
\end{array}\right.
$$

with the nonlinear characteristics

$$
g\left(x_{1}(t)\right)=\frac{1}{2}\left(m_{i}-m_{0}\right)\left(\left|x_{1}(t)+1\right|-\left|x_{1}(t)-1\right|\right)
$$

Choose parameters $m_{0}=-\frac{1}{7}, m_{1}=\frac{2}{7}, m_{2}=\frac{3}{7}, a_{1}=9, a_{2}=8, b_{1}=14.28, b_{2}=14.52, c_{1,1}=0.1$, $c_{1,2}=0.09, c_{2,1}=0.09, c_{2,2}=0.11$, and the constant time-delay $d_{1}=1, d_{2}=0.9$. It can be found that the corresponding system parameters are as follows:

$$
\begin{aligned}
\mathscr{A}_{i} & =\left[\begin{array}{ccc}
-a_{i} m_{i} & a_{i} & 0 \\
1 & -1 & 1 \\
0 & -b_{i} & 0
\end{array}\right], \mathscr{W}_{i}=\left[\begin{array}{ccc}
a_{i}\left(m_{i}-m_{0}\right) & 0 & 0 \\
0 & 0 & 0 \\
0 & 0 & 0
\end{array}\right], \quad \mathscr{D}=\left[\begin{array}{ccc}
1 & 0 & 0 \\
0 & 1 & 0 \\
0 & 0 & 1
\end{array}\right], \\
\mathscr{B}_{1, i} & =\left[\begin{array}{ccc}
-c_{1, i} & 0 & 0 \\
-c_{1, i} & 0 & 0 \\
2 c_{1, i} & 0 & -c_{1, i}
\end{array}\right], \mathscr{B}_{2, i}=\left[\begin{array}{ccc}
-c_{2, i} & 0 & 0 \\
-c_{2, i} & 0 & 0 \\
2 c_{2, i} & 0 & -c_{2, i}
\end{array}\right], \Gamma=\left[\begin{array}{cc}
-0.5 & 0.5 \\
1 & -1
\end{array}\right] .
\end{aligned}
$$

with $\eta_{1}\left(x_{1}(t)\right)=\frac{1}{2}\left(\left|x_{1}(t)+1\right|-\left|x_{1}(t)-1\right|\right)$ pertaining to sector $[0,1]$, and $\eta_{2}\left(x_{2}(t)\right)=\eta_{3}\left(x_{3}(t)\right)=0$. Choosing $\mathscr{C}_{1}=\mathscr{C}_{2}=\left[\begin{array}{lll}1 & 0 & 0\end{array}\right], \delta=0.5, \gamma=2, h_{m}=10^{-5}$. Table 1 presents the maximum values of $h_{M}$ for different $\alpha$ by using Theorem 1. It can be found that the choice of $\alpha$ has an impact on the value of $h_{M}$. Specifically, a larger $\alpha$ implies that the master-slave systems has faster synchronization speed, which corresponds to a smaller value of $h_{M}$.

Table 1: Maximum values of $h_{M}$ for different $\alpha$

\begin{tabular}{llllll}
\hline$\alpha$ & 0.1 & 0.2 & 0.3 & 0.4 & 0.5 \\
\hline$h_{M}$ & 0.2340 & 0.2050 & 0.1596 & 0.1347 & 0.1300 \\
\hline
\end{tabular}

Choosing $\alpha=0.3$ and $h_{M}=0.1596$, by solving the LMIs $(9)-(13)$, the gain matrix $\mathscr{K}_{1}$, $\mathscr{K}_{2}$ can be computed as:

$$
\begin{aligned}
& \mathscr{K}_{1}=\left[\begin{array}{lll}
4.9445 & 1.2194 & -4.3946
\end{array}\right]^{T}, \\
& \mathscr{K}_{2}=\left[\begin{array}{lll}
4.3834 & 1.2246 & -4.5036
\end{array}\right]^{T} .
\end{aligned}
$$

Assuming $x(t)=\left[\begin{array}{lll}0.2 & 0.3 & 0.2\end{array}\right]^{T}$ and $y(t)=\left[\begin{array}{lll}-0.3 & -0.1 & 0.4\end{array}\right]^{T}, t \in\left[\begin{array}{l}-1,0\end{array}\right]$ as the initial conditions of the master-slave systems. The Markovian jump mode and the corresponding state trajectories of the error system (3) without ASDC are displayed in Figure. 1(a). We can conclude that the error system (3) itself is 
unstable. On the basis of obtained ASDC by Theorem 1, Figure. 1(b) displays the Markovian jump mode and corresponding state trajectories of the error system (3). Furthermore, Figure. 1(c) displays the control input under the action of ASDC and the aperiodic sampling interval, from which one can get that the closed-loop system is stable. Figure. 1(d) shows the displayed chaotic behavior. Therefore, the ASDC can successfully achieve the master-slave systems exponential synchronization.

Furthermore, consider the Chua's circuit with a single time-delay and without Markovian jump parameters, that is, let $\mathscr{A}=\mathscr{A}_{1}, \mathscr{B}=\mathscr{B}_{1,1}, \mathscr{W}=\mathscr{W}_{1}, \mathscr{C}=\mathscr{C}_{1}, d=1$, and keep $\eta_{\sigma}\left(x_{\sigma}(t)\right), \delta, \gamma$ and $h_{m}$ unchanged. Based on Corollary 1, the specific numerical comparison is shown in Table 2. From comparison with [19] and [20], it can be seen that our method can enlarge the upper bound of the sampling period $h_{M}$. Therefore, the method developed in this paper significantly reduces the conservativeness.

Take it one step further, choosing $d=0, \alpha=0$, Table 3 shows the comparison of our result with some previous results when considering the Lur'e system without time-delay. It is clear that the results obtained in this paper are better than the existing results.

Table 2: Maximum values of $h_{M}$ for different $\alpha$

\begin{tabular}{llllll}
\hline$\alpha$ & 0.1 & 0.2 & 0.3 & 0.4 & 0.5 \\
\hline$[19]$ & 0.3247 & 0.2941 & 0.2658 & 0.2396 & 0.2154 \\
\hline$[20]$ & 0.4381 & 0.3919 & 0.3519 & 0.3164 & 0.2847 \\
\hline This paper & 0.4902 & 0.4880 & 0.4823 & 0.4743 & 0.4648 \\
\hline
\end{tabular}

Table 3: Maximum values of $h_{M}$ for $\alpha=0$ for different methods

\begin{tabular}{llllll}
\hline Methods & {$[16]$} & {$[17]$} & {$[18]$} & {$[19]$} & This Paper \\
\hline$h_{M}$ & 0.17 & 0.21 & 0.3914 & 0.3981 & 0.4540 \\
\hline
\end{tabular}

Example 2. Use the master-slave systems (1) and (2) with the following parameters:

$$
\begin{aligned}
& \mathscr{A}_{1}=\left[\begin{array}{ccc}
-1 & 0 & 0 \\
0 & -1 & 0 \\
0 & 0 & -1
\end{array}\right], \quad \mathscr{A}_{2}=\left[\begin{array}{ccc}
-1.1 & 0 & 0 \\
0 & -1.1 & 0 \\
0 & 0 & -1.1
\end{array}\right], \Gamma=\left[\begin{array}{cc}
-0.5 & 0.5 \\
1 & -1
\end{array}\right], \\
& \mathscr{W}_{1}=\left[\begin{array}{ccc}
1.2 & -1.6 & 0 \\
1.24 & 1 & 0.9 \\
0 & 2.2 & 1.5
\end{array}\right], \quad \mathscr{W}_{2}=\left[\begin{array}{ccc}
1.24 & -1.5 & 0 \\
1.2 & 0.9 & 1 \\
0 & 2.1 & 1.6
\end{array}\right], \quad \mathscr{C}_{1}=\mathscr{C}_{2}=\mathscr{D}=I \text {. }
\end{aligned}
$$

Based on above parameters, the system is reduced to a three neurons neural network with two mode. Moreover, the activation functions is $\eta_{\sigma}\left(x_{\sigma}(t)\right)=\frac{1}{2}\left(\left|x_{\sigma}(t)+1\right|-\left|x_{\sigma}(t)-1\right|\right), \sigma=1,2,3$, and thus $s_{1}=s_{2}=$ $s_{3}=1$. Denote $\delta=0.5, \gamma=2, h_{m}=10^{-5}$. Using Theorem 1 , the relationship between the maximum values of $h_{M}$ and $\alpha$ is displayed in Table 4. Choosing $\alpha=0.2$ and $h_{M}=0.3288$, by solving the LMIs (9)-(13), the 


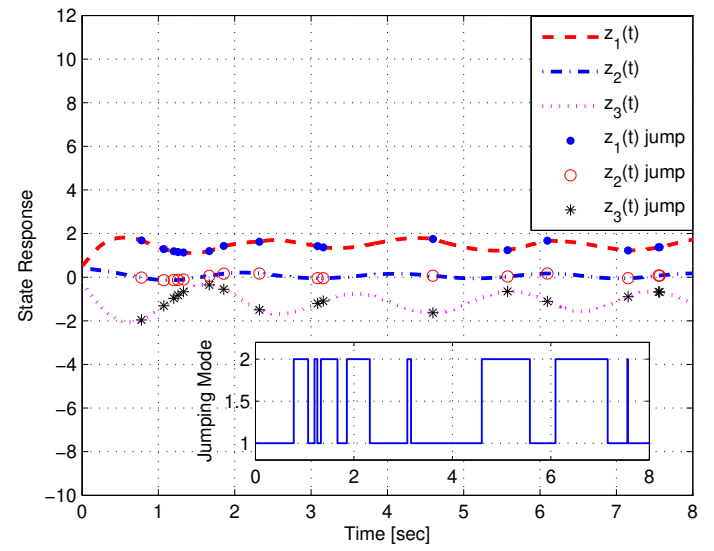

(a)

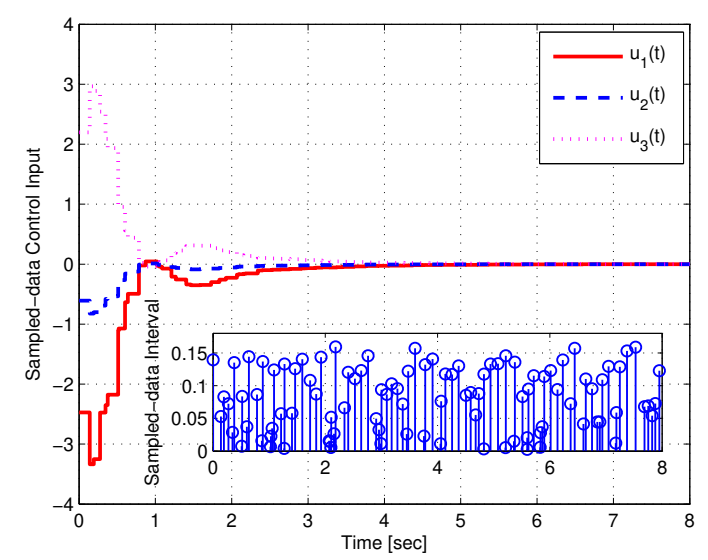

(c)

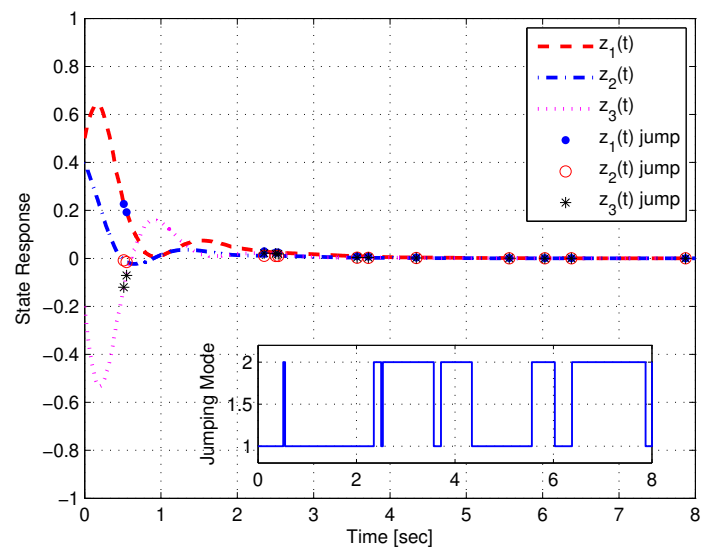

(b)
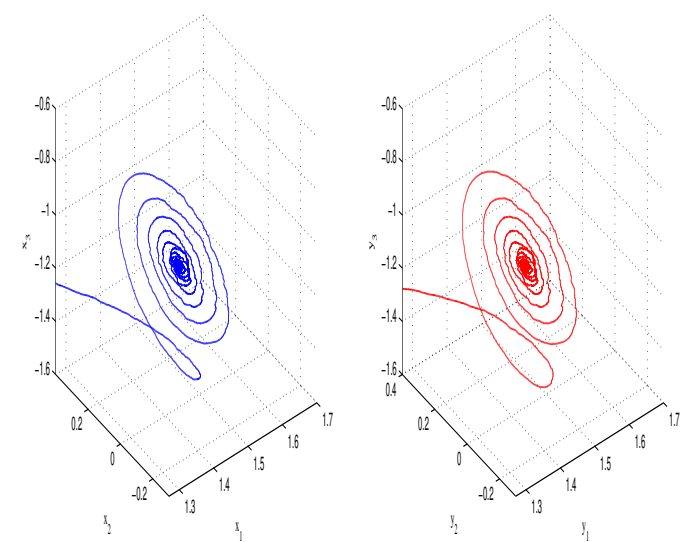

(d)

Figure 1: Open-loop state trajectory of the error system, closed-loop state trajectory of the error system, sampled-data control input and aperiodic sampled-data interval, chaotic behaviors of master system and slave system

gain matrix $\mathscr{K}_{1}, \mathscr{K}_{2}$ can be computed as:

$$
\mathscr{K}_{1}=\left[\begin{array}{ccc}
2.4299 & -1.0771 & 0.5392 \\
0.4121 & 1.4925 & 0.4596 \\
-1.0102 & 1.5581 & 1.1123
\end{array}\right], \mathscr{K}_{2}=\left[\begin{array}{ccc}
1.1822 & -0.2435 & -0.2448 \\
1.1023 & 0.8558 & 0.9797 \\
0.4682 & 0.4595 & 2.1018
\end{array}\right] .
$$

Assuming the initial conditions of the master-slave systems are $x(0)=\left[\begin{array}{lll}0.4 & 0.3 & 0.8\end{array}\right]^{T}$ and $y(0)=$ $\left[\begin{array}{lll}0.2 & 0.4 & 0.9\end{array}\right]^{T}$, respectively. The Markovian jump mode and the corresponding state trajectories of the error system (3) are displayed in Figure. 2(a) when there is no ASDC. We can get that the error system (3) itself is unstable. On the basis of the obtained ASDC, by Theorem 1, Figure. 2(b) displays the Markovian jump mode and the corresponding state trajectories of the error system (3). Furthermore, Figure. 2(c) displays the control input under the action of ASDC and aperiodic sampling interval, from which one can get that the closed-loop system is stable. Figure. 2(d) shows the displayed chaotic behavior. Therefore, the ASDC 


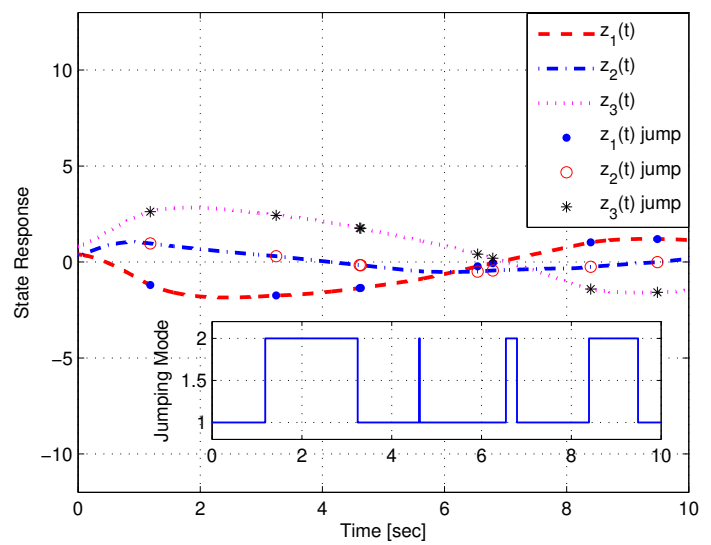

(a)

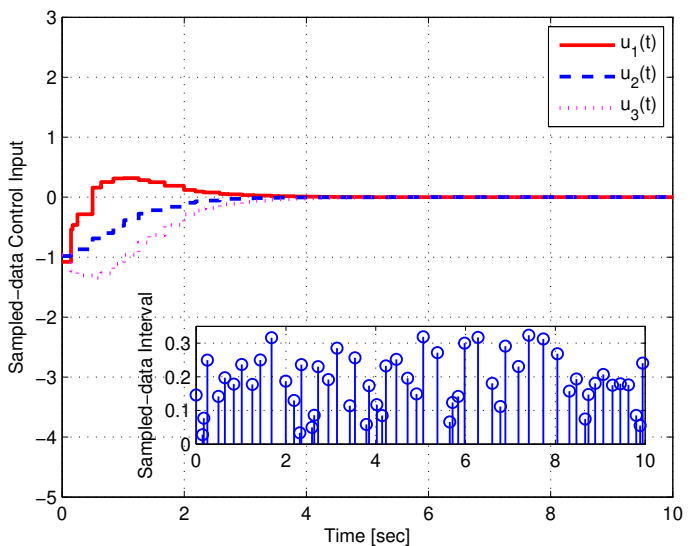

(c)

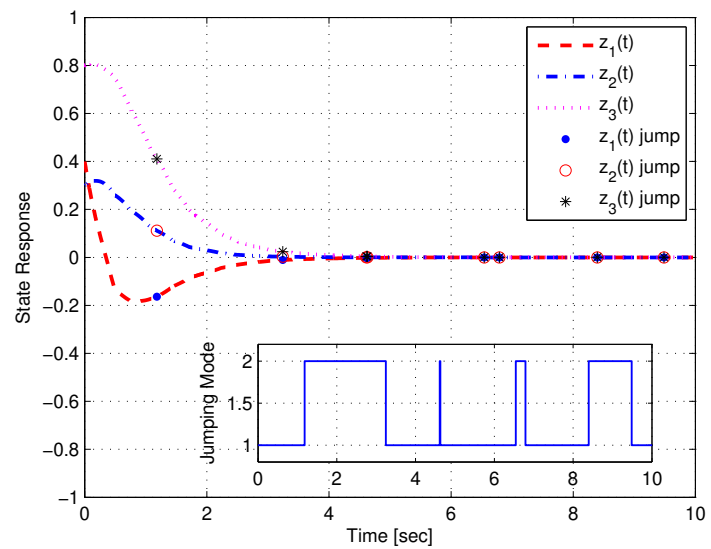

(b)


(d)

Figure 2: Open-loop state trajectory of the error system, closed-loop state trajectory of the error system, sampled-data control input and aperiodic sampled-data interval, chaotic behaviors of master system and slave system

can successfully stabilize the unstable error system. The master-slave systems exponential synchronization is achieved.

Furthermore, consider the neurons neural network without Markovian jump parameters, that is, let $\mathscr{A}=$ $\mathscr{A}_{1}, \mathscr{W}=\mathscr{W}_{1}, \mathscr{C}=\mathscr{C}_{1}$, and keep $\mathscr{D}, \eta_{\sigma}\left(x_{\sigma}(t)\right), \delta, \gamma$ and $h_{m}$ unchanged. Baed on Corollary 1, Table 5 shows the comparison of our result with some previous results, our method can enlarge the upper bound of the sampling period $h_{M}$. Therefore, the approach obtained in this article is effective and can ensure a lower conservativeness.

Table 4: Maximum values of $h_{M}$ for different $\alpha$

\begin{tabular}{llllll}
\hline$\alpha$ & 0.1 & 0.2 & 0.3 & 0.4 & 0.5 \\
\hline$h_{M}$ & 0.3395 & 0.3288 & 0.3171 & 0.3049 & 0.2919 \\
\hline
\end{tabular}


Table 5: Maximum values of $h_{M}$ for different methods

\begin{tabular}{llllll}
\hline Methods & {$[19]$} & {$[21]$} & {$[22]$} & {$[27]$} & This Paper \\
\hline$h_{M}$ & 0.3250 & 0.3818 & 0.9121 & 0.9213 & 0.9272 \\
\hline
\end{tabular}

\section{Conclusion}

The presented paper has proposed the mean square exponential synchronization criterion and the ASDC design method for the MJCLSs with multiple time delays. A novel construction of Lyapunov functionals is given by introducing mode-dependent single/double integral terms and mode-dependent sampled-state information. The estimation of the expectation of the solution plays a vital role in obtaining the exponential stability criterion in the mean square sense. On the basis of the stability criterion, a mode-dependent ASDC has been presented. Finally, a Chua's circuit and a neural network have been provided as numerical simulation examples to demonstrate the effectiveness of the raised methods.

\section{Acknowledgments}

This work was supported by the National Natural Science Foundation of China under Grants 61973199, 62003794, 62173214, the Shandong Provincial Natural Science Foundation ZR2020QF050, ZR2021MF003 and the Taishan Scholar Project of Shandong Province of China.

\section{Data Availability}

Data sharing not applicable to this article as no datasets were generated or analysed during the current study.

\section{Conflict of Interest}

The authors declare that they have no conflict of interest.

\section{References}

[1] J. H. Park, H. Shen, X. H. Chang, and T. H. Lee, "Recent Advances in Control and Filtering of Dynamic Systems with Constrained Signals," Switzerland: Springer, 2018, doi:10.1007/978-3-319-96202-3.

[2] J. H. Park, "Recent Advances in Control Problems of Dynamical Systems and Networks, Switzerland: Springer-Nature, 2020, doi: 10.1007/978-3-030-49123-9. 
[3] Y. Liu and S. M. Lee, "Synchronization of chaotic Lur'e systems using sampled-data PD control," Nonlinear Dyn., vol. 85, no.3, pp. 981-992, Jul. 2016.

[4] W. He, F. Qian, Q. L. Han, and J. Cao, "Synchronization error estimation and controller design for delayed Lur'e systems with parameter mismatches," IEEE Trans. Neural Netw. Learn. Syst., vol. 23, no. 10, pp. 1551-1563, Oct. 2012.

[5] J. Song, Y. Niu, and J. X, "An event-triggered approach to sliding mode control of Markovian jump Lur'e systems under hidden mode detections," IEEE Trans. Syst., Man, Cybern., Syst., vol. 50, no. 4, pp. 1514-1525, Apr. 2020.

[6] C. A. C. Gonzaga, and O. L. V. Costa, "Stochastic stabilization and induced L2-gain for discrete-time Markov jump Lur'e systems with control saturation," Automatica, vol. 50, no. 9, pp. 2397-2404, Sep. 2014.

[7] B. Zhang, and Y. Song, "Efficient model predictive control for Markovian jump systems with Lur'e nonlinear term: A dual-mode control scheme," Int. J. Robust Nonlin. Control, early access, Oct. 2021, doi: $10.1002 /$ rnc. 5844 .

[8] J. Huang, P. Wang, Z. Han, and X. Cai, "Observer design for the Lur'e differential inclusion system with Markovian jumping parameters," Int. J. Syst. Sci., vol. 44, no. 12, pp. 2338-2348, Dec. 2013.

[9] Z. G. Wu, P. Shi, H. Su, and J. Chu, "Exponential stabilization for sampled-data neural-network-based control systems," IEEE Trans. Neural Netw. Learn. Syst., vol. 25, no. 12, pp. 2180-2190, Dec. 2014.

[10] Z. G. Wu, P. Shi, H. Su, and J. Chu, "Local synchronization of chaotic neural networks with sampled-data and saturating actuators," IEEE Trans. Cybern., vol. 44, no. 12, pp. 2635-2645, Dec. 2014.

[11] T. Yang, Z. Wang, X. Huang, J. Xia, and G. Chen, "Aperiodic sampled-data controller design for stochastic Markovian jump systems and its application," Int. J. Robust Nonlin. Control, vol. 31, no. 14, pp. 6721-6739, 2021.

[12] G. Chen, C. Fan, J. Sun, and J. Xia, "Mean square exponential stability analysis for Ito stochastic systems with aperiodic sampling and multiple time-delays," IEEE Trans. Automatic Control, early access, Apr. 2021, doi.10.1109/TAC.2021.3074848.

[13] G. Chen, C. Fan, J. Lam J. Sun, and J. Xia, "Aperiodic sampled-data controller design for switched Ito stochastic Markovian jump systems," Systems 8$\}$ Control Letters, vol. 157, no. 105301, pp. 1-11, Nov. 2021. 
[14] G. Chen, J. Xia, J. H. Park, H. Shen and G. Zhuang, "Robust sampled-data control for switched complex dynamical networks with actuators saturation," IEEE Trans. Cybern., early access, Apr. 2021, doi: 10.1109/TCYB.2021.3069813.

[15] L. M. Pecora and T. L. Carroll, "Synchronization in chaotic systems," Phys. Rev. Lett., vol. 64, no. 8, pp. 821-824, Feb. 1990.

[16] J. Lu and D. J. Hill, "Global asymptotical synchronization of chaotic Lur'e systems using sampled data: A linear matrix inequality approach," IEEE Trans. Circuits Syst. II-Exp. Briefs, vol. 55, no. 6, pp. 586-590, Jun. 2008.

[17] C. -K. Zhang, Y. He, and M. Wu, "Improved global asymptotical synchronization of chaotic Lur'e systems with sampled-data control," IEEE Trans. Circuits Syst. II Exp. Briefs, vol. 56, no. 4, pp. 320-324, Mar. 2009 .

[18] W. -H. Chen, Z. Wang, and X. Lu, "On sampled-data control for master-slave synchronization of chaotic Lur'e systems," IEEE Trans. Circuits Syst. II-Exp. Briefs, vol. 59, no. 8, pp. 515-519, Aug. 2012.

[19] Z. -G. Wu, P. Shi, H. Su, and J. Chu, "Sampled-data synchronization of chaotic Lur'e systems with time delays," IEEE Trans. Neural Netw. Learn. Syst., vol. 24, no. 3, pp. 410-421, Jan. 2013.

[20] H. -B. Zeng, Ju H. Park, S. P. Xiao, and Y. Liu, "Further results on sampled-data control for master-slave synchronization of chaotic Lur'e systems with time delay," Nonlinear Dyn., vol. 82, no. 1-2, pp. 851-863, Oct. 2015.

[21] K. Shi, X. Liu, and H. Zhu, "Novel integral inequality approach on master-slave synchronization of chaotic delayed Lur'e systems with sampled-data feedback control," Nonlinear Dyn., vol. 83, no. 3, pp. 1259-1274, Feb. 2016.

[22] C. Hua, C. Ge, and X. Guan, "Synchronization of chaotic Lur'e systems with time delays using sampleddata control," IEEE Trans. Neural Netw. Learn. Syst., vol. 26, no. 6, pp. 1214-1221, Jun. 2015.

[23] C. Ge, B. Wang, J. H. Park, and C. Hua, "Improved synchronization criteria of Lur'e systems under sampled-data control," Nonlinear Dyn., vol. 94, no. 9, pp. 2827-2839, Dec. 2018.

[24] J. Cao, R. Sivasamy, and R. Rakkiyappan, "Sampled-data $H \infty$ synchronization of chaotic Lur'e systems with time delay," Circuits Syst. Signal Process., vol. 35, no. 3, pp. 811-835, Mar. 2016.

[25] Y. Zhu, W. X. Zheng and D. Zhou, "Quasi-synchronization of discrete-time Lur'e-type switched systems with parameter mismatches and relaxed PDT constraints," IEEE Trans. Cybern., vol. 50, no. 5, pp. 2026-2037, May 2020. 
[26] Z. Tang, J. H. Park, Y. Wang and J. Feng, "Distributed impulsive quasi-synchronization of Lur'e networks with proportional delay," IEEE Trans. Cybern., vol. 49, no. 8, pp. 3105-3115, Aug. 2019.

[27] K. Shi, Y. Tang, X. Liu, and S. Zhong, "Non-fragile sampled-data robust synchronization of uncertain delayed chaotic Lur'e systems with randomly occurring controller gain fluctuation," ISA Trans., vol. 66, pp. 185-199, Jan. 2017.

[28] R. Zhang, D. Zeng, and S. Zhong, "Novel master-slave synchronization criteria of chaotic Lur'e systems with time delays using sampled-data control," J. Frankl. Inst., vol. 354, no.12, pp. 4930-4954, Aug. 2017.

[29] R. Zhang, D. Zeng, X. Liu, S. Zhong, and K. Shi, "A new method for quantized sampled-data synchronization of delayed chaotic Lur'e systems," Appl. Math. Model., vol. 70, no.12, pp. 471-489, Jun. 2019.

[30] X. Yang, J. Cao, and J. Lu, "Synchronization of Markovian coupled neural networks with nonidentical node-delays and random coupling strengths," IEEE Trans. Neural Networks Learn. Syst., vol. 23, no. 1, pp. 60-71, Jan. 2012.

[31] X. Yang, Q. Song, J. Cao, and J. Lu, "Synchronization of coupled Markovian reaction-diffusion neural networks with proportional delays via quantized control," IEEE Trans. Neural Networks Learn. Syst., vol. 3, no. 3, 951-958, Mar. 2019.

[32] H. Zhang, J. Wang, Z. Wang, and H. Liang, "Sampled-data synchroniza-tion analysis of Markovian neural networks with generally incomplete transition rates," IEEE Trans. Neural Netw. Learn. Syst., vol. 28, no. 3, pp. 740-752, Mar. 2017.

[33] J. Wang, H. Zhang, Z. Wang, and Z. Liu, "Sampled-data synchronization of Markovian coupled neural networks with mode delays based on mode-dependent LKF," IEEE Trans. Neural Netw. Learn. Syst., vol. 28, no. 11, pp. 2626-2637, Nov. 2017.

[34] G. Chen, J. Xia, Ju H. Park, H. Shen, and G. Zhuang, "Sampled-data synchronization of stochastic Markovian jump neural networks with time-varying delay," IEEE Trans. Neural Netw. Learn. Syst., early access, Feb. 2021. doi: 10.1109/TNNLS.2021.3054615.

[35] H. Shen, X. Wang, J. Wang, J. Cao, and L. Rutkowski, "Robust composite $H \infty$ synchronization of Markov jump reaction-diffusion neural networks via a disturbance observer-based method," IEEE Trans. Cybern., early access, Aug. 2021. doi: 10.1109/TCYB.2021.3087477.

[36] H. Shen, X. Hu, J. Wang, J. Cao, and W. Qian, "Non-fragile $H \infty$ synchronization for Markov jump singularly perturbed coupled neural networks subject to double-layer switching regulation," IEEE Trans. Neural Netw. Learn. Syst., early access, Sep. 2021. doi: 10.1109/TNNLS.2021.3107607. 
[37] W. Kang, J. Cheng, X. Zhou, J. Cao, and H. Wang, "Asynchronous quantized control of Markovian switching Lur'e systems with event-triggered strategy," J. Frankl. Inst. vol. 358, no. 3, pp. 1984-1988, Feb. 2021.

[38] R. Nie, S. He, F. Liu, X. Luan, and H. Shen, "HMM-Based asynchronous controller design of Markovian jumping Lur'e systems within a finite-time interval," IEEE Trans. Syst., Man, Cybern., Syst., vol. 51, no. 11, pp. 6885-6891, Nov. 2021.

[39] Q. Li, X. Liu, Q. Zhu, S. Zhong, and J. Cheng, "Stochastic synchronization of semi-Markovian jump chaotic Lur'e systems with packet dropouts subject to multiple sampling periods," J. Frankl. Inst., vol. 356, no. 13, pp. 6899-6925, Sep. 2019.

[40] H. Huang, G. Feng, and X. Chen, "Stability and stabilization of Markovian jump systems with time delay via new lyapunov functionals," IEEE Trans.Circuits Syst. I-Reg. Pap., vol. 59, no. 10, pp. 2413-2421, Oct. 2012.

[41] H. -B. Zeng, Y. He, M. Wu, and J. She, "New results on stability analysis for systems with discrete distributed delay," Automatica, vol. 60, pp. 189-192, Oct. 2015.

[42] H. -B. Zeng, K. L. Teo, and Y. He, "A new looped-functional for stability analysis of sampled-data systems," Automatica, vol. 82, pp. 328-331, Aug. 2017. 